\title{
Parallel Mitral and Tufted Cell Pathways Route Distinct Odor Information to Different Targets in the Olfactory Cortex
}

\author{
Kei M. Igarashi, ${ }^{1,3 *}$ Nao Ieki, ${ }^{1,2 *}$ Myungho An, ${ }^{1,2}$ Yukie Yamaguchi, ${ }^{1}$ Shin Nagayama, ${ }^{4}$ Ko Kobayakawa, ${ }^{5}$ \\ Reiko Kobayakawa, ${ }^{5}$ Manabu Tanifuji, ${ }^{3}$ Hitoshi Sakano, ${ }^{5}$ Wei R. Chen, ${ }^{4}$ and Kensaku Mori ${ }^{1,2}$ \\ ${ }^{1}$ Department of Physiology, Graduate School of Medicine, The University of Tokyo, and ${ }^{2}$ Japan Science and Technology Agency, CREST, Tokyo 113-0033, \\ Japan, ${ }^{3}$ Laboratory for Integrative Neural Systems, RIKEN Brain Science Institute, Saitama 351-0198, Japan, ${ }^{4}$ Department of Neurobiology and Anatomy, \\ University of Texas Medical School at Houston, Houston, Texas 77030, and ${ }^{5}$ Department of Biophysics and Biochemistry, Graduate School of Science, The \\ University of Tokyo, Tokyo 113-0032, Japan
}

Odor signals are conveyed from the olfactory bulb to the olfactory cortex (OC) by mitral cells (MCs) and tufted cells (TCs). However, whether and how the two types of projection neuron differ in function and axonal connectivity is still poorly understood. Odor responses and axonal projection patterns were compared between MCs and TCs in mice by visualizing axons of electrophysiologically identified single neurons. TCs demonstrated shorter onset latency for reliable responses than MCs. The shorter latency response of TCs was maintained in a wide range of odor concentrations, whereas MCs responded only to strong signals. Furthermore, individual TCs projected densely to focal targets only in anterior areas of the OC, whereas individual MCs dispersedly projected to all OC areas. Surprisingly, in anterior OC areas, the two cell types projected to segregated subareas. These results suggest that MCs and TCs transmit temporally distinct odor information to different OC targets.

\section{Introduction}

The processing of different aspects of sensory information in parallel pathways is a basic principle in the visual, auditory, and somatosensory systems (Konishi, 2003; Petersen, 2007; Nassi and Callaway, 2009). The olfactory system processes a variety of odor information that includes quality and concentration of inhaled odorants as well as temporal change in odor signals. In the mammalian olfactory bulb (OB), two major types of projection neuron, mitral cells (MCs) and tufted cells (TCs), receive the olfactory sensory inputs within glomeruli and transmit the odor information to various areas of the olfactory cortex (OC) (Shepherd et al., 2004; Mori and Sakano, 2011). However, the differences between MCs and TCs in terms of olfactory information

Received Jan. 11, 2012; revised March 3, 2012; accepted April 25, 2012.

Author contributions: K.M.I., S.N., W.R.C., and K.M. designed research; K.M.I., N.I., M.A., and Y.Y. performed research; K.K., R.K., and H.S. contributed unpublished reagents/analytic tools; K.M.I., N.I., M.T., and K.M. analyzed data; K.M.I., N.I., and K.M. wrote the paper.

This work was supported by Ministry of Education, Culture, Sports, Science and Technology of Japan Grants 17023012 and 18100004 (K.M.). K.M.I. was a Japan Society for the Promotion of Science Postdoctoral Research Fellow (19-3183). S.N. was supported by multiple NIH grants (NIH Grants DC010057 and DC009666). We thank Drs. Yoshikazu Isomura for technical advice on juxtacellular recording, Takahiro Furuta for technical advice on the staining protocol, and Karel Svoboda, Gordon M. G. Shepherd, Leopoldo Petreanu, and Takaki Komiyama for providing scripts for morphological analyses. We also thank Dr. Yoshihiro Yoshihara and members of the Sakano, Chen, Tanifuji, and Mori Laboratories for support and comments on this manuscript

The authors declare no competing financial interests.

*K.M.I. and N.I. contributed equally to this work.

Correspondence should be addressed to either of the following: Dr. Kei M. Igarashi, Kavli Institute for Systems Neuroscience and Centre for the Biology of Memory, Norwegian University of Science and Technology, 7491 Trondheim, Norway, E-mail: kei.igarashi@ntnu.no; or Dr. Kensaku Mori, Department of Physiology, Graduate School of Medicine, The University of Tokyo, 7-3-1 Hongo, Bunkyo-ku, Tokyo 113-0033, Japan, E-mail: moriken@m.u-tokyo.ac.jp.

DOI:10.1523/JNEUROSCI.0154-12.2012

Copyright $\odot 2012$ the authors $\quad 0270-6474 / 12 / 327970-16 \$ 15.00 / 0$ processing and the axonal targets within each area of the OC are still poorly understood (Mori and Sakano, 2011).

First, do MCs and TCs differ in their manner of responding to odor inhalation? Previous physiological studies have shown that MCs have finer odorant receptive ranges, whereas TCs show burst spike responses with higher firing frequency and stronger phase-locking to respiration cycles (Nagayama et al., 2004; Griff et al., 2008). However, recent behavioral studies demonstrated that rodents extract sufficient odor information within the first respiration of odor sampling (Uchida and Mainen, 2003; Abraham et al., 2004; Rinberg et al., 2006). Thus, understanding the temporal profile of $\mathrm{OB}$ neuron odor responses within the time course of one respiration cycle has become critical (Bathellier et al., 2008; Cury and Uchida, 2010; Junek et al., 2010; Carey and Wachowiak, 2011; Wachowiak, 2011), but it is still unclear whether MCs and TCs differ in signal timing with regard to the inspiration of odorants.

Second, do MCs and TCs differ in their axonal targets in each area of the OC? Since both MCs and TCs project axons to the OC, odor information encoded by each type of odorant receptor is conveyed from the OB to the OC via axons of both cell types. Gross anatomical studies previously indicated that TCs send axons only to anterior areas of the OC, including the anterior olfactory nucleus (AON), anterior piriform cortex (APC), and olfactory tubercle, whereas MCs project to both the anterior areas and additional posterior OC areas (Haberly and Price, 1977; Scott, 1981; Luskin and Price, 1982). However, it remains unknown whether these two cell types project to shared or segregated targets within the anterior three OC areas. This is a crucial question if MCs and TCs carry temporally distinct patterns of spike signals. Our previous investigation using tracer injection 
into a single glomerulus revealed distinct tendency in the directions of axon branches from these tract fibers, but could not conclude about distinction of terminal axon bushes (Nagayama et al., 2010). The distinction between MCs and TCs in the anterior OC areas still remains poorly explored in recent highresolution anatomical studies (Ghosh et al., 2011; Miyamichi et al., 2011; Sosulski et al., 2011).

To resolve these two questions, we adopted a juxtacellular single-cell recording (Pinault, 1996) in combination with an amplifying axon labeling (Furuta et al., 2009) and compared between MCs and TCs for their temporal profiles of responses to two specific odorants and axon terminal bushes over whole areas of the OC.

\section{Materials and Methods}

Animal surgery. All experiments were performed in accordance with the guidelines of the Physiological Society of Japan and the animal experiment committee of the University of Tokyo. Experiments were performed on 64 male adult mice (C57BL/6; 11-15 weeks of age; 20-30 g; Japan SLC). Animals were first injected with medetomidine $(0.5 \mathrm{mg} / \mathrm{kg})$ for induction of anesthesia. After $15 \mathrm{~min}$, ketamine $(22.5 \mathrm{mg} / \mathrm{kg})$ was injected as deep anesthesia for preparation surgery (heart rate, 150-200/ $\mathrm{min}$ ). For anesthesia during recording, we used pentothal sodium, which enabled us to perform the optical imaging, physiological recording, and animal recovery after recordings to trace their axons. At $2.5 \mathrm{~h}$ after ketamine injection when the effect of ketamine had decayed (heart rate, $300 / \mathrm{min})$, pentothal sodium $(25 \mathrm{mg} / \mathrm{kg}$ ) was injected to maintain light anesthesia during recordings (heart rate, $300-350 / \mathrm{min}$ ). Adequate anesthesia was confirmed by the lack of withdrawal responses to forelimb pinching. Additional doses of pentothal sodium were given when necessary. Animals were placed in a stereotaxic apparatus (SR-6N; Narishige). Body temperature was maintained at $37.5^{\circ} \mathrm{C}$ using a homeothermic heatpad system (MK-900; Muromachi). Respiratory rhythms (plethysmograph) were detected using a piezoelectric transducer (MLT 1010; ADInstruments).

Odor stimulation. The compounds 2,4,5-trimethylthiazoline (TMT) (Contech) and 2-methylbutyric acid (2MBA) (Tokyo Kasei) were used for odor stimulation in the optical imaging and electrophysiological experiments. Odorants were delivered using a computer-controlled custom-built olfactometer as described previously (Yoshida and Mori, 2007). The duration of each odor application was $6 \mathrm{~s}$ for optical imaging and $4 \mathrm{~s}$ for electrophysiological recordings, with at least $30 \mathrm{~s}$ interstimulus intervals to avoid habituation.

For the concentration series, TMT and 2MBA were diluted with mineral oil (Sigma-Aldrich) to concentrations of $1.0 \times 10^{-5}, 3.3 \times 10^{-5}$, $1.0 \times 10^{-4}, 3.3 \times 10^{-4}, 1.0 \times 10^{-3}, 1.0 \times 10^{-2}$, and $1.0 \times 10^{-1}(\mathrm{v} / \mathrm{v})$. The performance of the olfactometer was tested for concentration precision using a gas chromatograph (Hitachi; G-3000) fitted with a flame ionization detector. The relationship between output vapor concentrations from the olfactometer and the concentrations of the loaded solution were calibrated from five concentrations with linear regression $(r=0.99 ; p=0.0011)$. Vapor concentrations were then computed from the calibration line as $1.5,3.2,7.1,15,35,170$, and 830 parts per billion (ppb) for $1.0 \times 10^{-5}, 3.3 \times 10^{-5}, 1.0 \times 10^{-4}, 3.3 \times 10^{-4}, 1.0 \times$ $10^{-3}, 1.0 \times 10^{-2}$, and $1.0 \times 10^{-1}$ solutions, respectively. Concentrations of 2MBA were also calibrated using the gas chromatograph as 0.79 , $2.8,10,35,126,447,1585$, and $19,950 \mathrm{ppb}$ for $1.0 \times 10^{-5}, 3.3 \times 10^{-5}$, $1.0 \times 10^{-4}, 3.3 \times 10^{-4}, 1.0 \times 10^{-3}, 3.3 \times 10^{-3}, 1.0 \times 10^{-2}$, and $1.0 \times$ $10^{-1}$ solutions, respectively.

Optical imaging of intrinsic signals. Detailed procedures for the optical imaging have been described previously (Uchida et al., 2000). Images were analyzed using IDL (Research Systems) and MATLAB (MathWorks) software. A differential image was obtained by dividing the magnitude of signals acquired during odorant stimulation (frames 9-12) by those acquired before stimulation (frames 1-4). A Gaussian spatial filter was used to eliminate nonspecific global darkening and highfrequency noise of the differential image (cutoff frequencies, $\sigma=70.0 / \mathrm{mm}$ for the high cutoff and $\sigma=0.4 / \mathrm{mm}$ for the low cutoff). To achieve a better signal-to-noise ratio, these filtered images were averaged for at least five trials. The pixel intensities of the images with stimulus were compared by $t$ test with those with blank (pure air) stimulus. TMT- or 2MBA-responsive glomeruli ( $p<0.01, t$ test) were overlaid on the image of the blood vessels and used for the electrophysiological experiments.

Electrophysiological recordings and single-cell labeling. Single-unit recording and single-neuron labeling were performed using the juxtacellular recording technique (Pinault, 1996). A small area $(1 \times 1 \mathrm{~mm})$ of the bone above the TMT- or 2MBA-responsive glomeruli was removed with fine forceps. A glass micropipette (tip diameter, 0.5-1.0 $\mu \mathrm{m}$; impedance, $40-80 \mathrm{M} \Omega$ ) filled with $0.1 \mathrm{~m}$ potassium acetate, $\mathrm{pH} 7.5$, containing $12 \%$ biotinylated dextran amine (BDA-3000) (Invitrogen) was lowered near the responsive glomeruli. MCs and TCs responsive to TMT or 2MBA were searched randomly from a $300 \times 300 \mu \mathrm{m}$ area that spanned $0-300$ $\mu \mathrm{m}$ posteriorly and medially from the peak of the intrinsic signal. Once a single unit was extracellularly recorded and identified, the micropipette was then slowly advanced toward the neuron until the spike amplitude became $>1 \mathrm{mV}$. Odor-evoked activities (more than five trials for each odor concentration) were recorded with an amplifier (MEZ-8300; Nihon Kohden), filtered (150-10 kHz; AB-651J; Nihon Kohden), and stored in the computer via an $\mathrm{AD}$ converter with the Spike2 software package (Cambridge Electronic Design).

After the recording, neurons were electroporated to inject BDA by passage of positive current pulses (0.5-1.5 nA; 400 ms duration; 50\% duty cycle) through the bridge circuit of the amplifier. The firing of the recorded neuron was continuously monitored during and after the current injection to ensure that the neuron was steadily held throughout the session. For axonal tracing in the OC, the current injection was continued for $>30 \mathrm{~min}$. Only one neuron was labeled per each olfactory bulb in samples for axon reconstruction experiments. Since TMT- and 2MBAresponsive neurons are located distant enough $(\sim 1 \mathrm{~mm})$ to be distinguished, either or both the TMT and 2MBA-responsive neurons were labeled in experiments without axon reconstruction.

Neuronal and respiration data analysis. Analyses of respiration and spike responses were performed off-line using the Spike2 and MATLAB software packages.

Detection of respiration cycles. To establish the fidelity of the respiration timing measurements, pilot experiments were performed ( $n=3$ mice) to compare signals from piezoelectric transducer (plethysmograph) and temperature sensor (thermocouple) inserted directly in the nostril (1). Onsets of inhalation $\left(\mathrm{OI}_{\mathrm{T}}\right)$ were first determined from troughs (start of cooling) in the thermocouple signals. Inhalation timings were next detected by plethysmograph using peaks in the derivative of plethysmograph (i.e., acceleration of chest movement), since these peaks $\left(\mathrm{P}_{\mathrm{dP}}\right)$ were closer to $\mathrm{OI}_{\mathrm{T}}$ than peaks in the raw signal. Deflection between $\mathrm{OI}_{\mathrm{T}}$ and $\mathrm{P}_{\mathrm{dP}}$ was $28 \pm 0.8^{\circ}$ in respiration cycles $(n=2000$ respirations, 3 mice). Inhalation onsets were thus defined as the time point $28^{\circ}$ ahead of $\mathrm{P}_{\mathrm{dP}}$ in the following experiments. Exhalation onsets and offsets were also assessed by comparing thermocouple and plethysmograph signals, and defined as time points $3^{\circ}$ ahead and $80^{\circ}$ after troughs in plethysmograph signals, respectively. Temporal delay from solenoid valve onset to odor arrival at the tip of the outlet nozzle $\left(D_{\mathrm{VO}}\right)$ was calculated as $393 \mathrm{~ms}$ from a dead volume of tubings and the flow rate of the olfactometer. Thus, the odor arrival time was estimated to be $393 \mathrm{~ms}$ after the solenoid valve onset time. Onset of the first respiration just after the odor arrival time was defined as the onset of actual odor stimulation.

Peristimulus time histograms. Response spikes evoked by odor stimulation were aligned at first inhalation onsets just after the odor stimuli onsets for multiple trials. Peristimulus time histograms were then calculated using a Gaussian filter (SD, $25 \mathrm{~ms}$ ).

Peristimulus phase histograms. To clearly demonstrate the spike responses and respiration phases (inspiration-expiration) evoked by odor concentration series, peristimulus phase histograms were used. Inhalation onsets were defined as $0^{\circ}$ in each respiration cycle. In each respiration, spikes were binned ( 1 respiration $=20$ bins), and the firing rate (in spikes/second) was calculated for each bin using the actual duration (in seconds) of the bin. Respiration cycles were aligned by the cycle number 
after the odor stimulus onset. Bins were then averaged across trials and processed using a Gaussian filter $\left(\mathrm{SD}, 36^{\circ}\right)$.

Reliability index. To assess how informative the firing response of a neuron to a particular stimulus was compared with spontaneous firings, receiver operating characteristic (ROC) analysis was used, which has been widely used for comparing neuronal responses to different stimuli (Cury and Uchida, 2010). ROC curves were plotted for true-positive rate as a function of false-positive rate for mean firing rates between odor and blank trials. The area under the ROC curve (auROC) was used as a measure of the reliability of the stimulus response compared with spontaneous firing. An auROC value of 0.5 indicates that the stimulus cannot be detected; an auROC value of 1 corresponds to a stimulus that can be perfectly detected. Then, a metric, "reliability index," defined as RI = $2^{*}$ (auROC -0.5$)$, was used, where RI of 0 indicates that the response is not informative, and RI of 1 indicates that it is perfectly informative. ROC analysis was performed using 10, 20, 40, 80, 160, and $320 \mathrm{~ms}$ bins. For each bin, the statistical significance of RI compared with the RI value of 0 was assessed with a $z$ test $(p<0.05)$. Statistically significant bins that emerged earliest after the first inhalation onsets from odor stimulus onset $\left(\mathrm{RI}_{\mathrm{ES}}\right)$ were then determined. Cells that demonstrated $\mathrm{RI}_{\mathrm{ES}}$ during the first respiration after $830 \mathrm{ppb}$ TMT or $19,950 \mathrm{ppb} 2 \mathrm{MBA}$ stimulation were defined as TMT- or 2MBA-responsive cells, respectively, and cells without $\mathrm{RI}_{\mathrm{ES}}$ were discarded from our cell database. Note that cells can use any temporal bin size for encoding $\mathrm{RI}_{\mathrm{ES}}$. For assessing spontaneous firings, spike firings to blank mineral oil stimulations or prestimulus firing during a respiration that precede 10 respirations before stimulus onset were used. No difference was observed in spike firings between mineral oil stimulations and prestimulus firings ( $n=8$ cells).

Tracer visualization. After a survival period of $3 \mathrm{~d}$, the animals were administered an overdose of anesthetic and perfused transcardially with saline followed by a fixative containing $4 \%$ paraformaldehyde and $0.5 \%$ glutaraldehyde in phosphate buffer (PB) $(0.1 \mathrm{M}), \mathrm{pH}$ 7.4. Brains were embedded in egg yolk, postfixed overnight, and cryoprotected in $30 \%$ sucrose in PB for 2-3 d. Consecutive parasagittal sections (50 $\mu \mathrm{m}$ thickness) were obtained from individual brain hemispheres using a cryostat (HM500-M; Carl Zeiss). Using the free-floating method, sections were processed for the visualization of BDA according to the avidin-biotinperoxidase complex (ABC) histochemical protocol (ABC Elite; Vector Laboratories). The signal was then amplified using a tyramide signal amplification protocol (Furuta et al., 2009). The tracer was revealed by reaction with $0.05 \%$ diaminobenzidine, $0.5 \%$ nickel sulfate, and $0.007 \%$ hydrogen peroxide in $0.05 \mathrm{M}$ Tris buffer, $\mathrm{pH}$ 7.6, for $5 \mathrm{~min}$. Sections were mounted, dried overnight, and counterstained with Nuclear Fast Red (Vector Laboratories). Cells were defined as MCs if their somas were in the mitral cell layer. TCs were defined if their somas were located in the external plexiform layer. TCs were further classified into external tufted cells (eTCs), middle tufted cells (mTCs), and internal tufted cells (iTCs), whose somata were located at the border between the external plexiform layer (EPL) and glomerular layer, in the superficial two-thirds of the EPL, and in the deeper one-third of the EPL, respectively (Macrides and Schneider, 1982; Shepherd et al., 2004).

Three-dimensional reconstruction. Mitral and tufted cells were threedimensionally reconstructed using a computer-assisted system (Neurolucida; MBF Bioscience) according to the manufacturer's instructions. Axons, dendrites, cell bodies, brain surfaces, and cell layers in each region within the olfactory bulb and olfactory cortex were traced. In samples with injections of the tracer for $>30 \mathrm{~min}$, almost all axons were continuously traced up to the lateral entorhinal cortex, suggesting that nearly complete axons of mitral and tufted cells were successfully visualized. To simplify the tracing procedures, only axon collaterals were traced to assess the projection patterns of individual neurons, and bouton structures were not registered. A single mitral cell usually required $\sim 2$ months for three-dimensional (3-D) reconstruction.

Morphological data analysis. The tracing output from Neurolucida was imported into MATLAB for further analyses. To place neurons in identical coordinates and minimize the distortions originating from sectioning and reconstruction, reconstructed neurons were linearly transformed and fitted into reference brain coordinates. A reference brain was first constructed by reconstructing the reference mouse brain sections in the
Allen Brain Atlas (http://mouse.brain-map.org/). Three landmarks for transformations were used: the end of the lateral olfactory tract (eLOT), the dorsomedial edge of the pars externa in the anterior olfactory nucleus (AONpEd), and the ventromedial edge of the pars externa in the anterior olfactory nucleus (AONpEv). These landmarks were selected because (1) they were distributed at the periphery of the tufted-targeted areas and (2) they could be uniquely defined and were unaffected by rotation of the brain. The coordinate system was defined as giving the AONpEv coordinates of $[0,0,0]$ in the $[x$ (posterior), $y$ (dorsal), and $z$ (lateral) $]$ directions (in micrometers). Individual sample brains containing a neuron were then fitted to the reference brain with regard to the above landmarks using affine (linear) transformation (translation, rotation, scaling, and shear). For the ventrolateral-view presentation, transformed brains were then rotated $-60^{\circ}$ around the $x$-axis, $30^{\circ}$ around the $y$-axis, and $-25^{\circ}$ around the $z$-axis. This projection has minimum overlap of axonal projections in the olfactory cortex for samples stained in this study, except for the tenia tecta. For the tenia tecta, axons were presented in a medial view.

Axon and dendrite density maps. The densities of axons and dendrites were calculated per $100 \times 100 \mu \mathrm{m}$ bin (Shepherd et al., 2005). This calculation was performed after individual cells were overlaid using the ventrolateral-view fitted coordinates for olfactory cortex axons, and after they were aligned by the position of the soma for olfactory bulb axons and dendrites. The density is represented on a color scale created using three rounds of interpolation between bins.

Axon and dendrite length. In the quantitative analysis of olfactory bulb local axons, axons that extended to the olfactory cortex were excluded from their last branching point. For olfactory cortex axons, reconstructed axons were detached into those of individual regions of the olfactory cortex with reference to the position of reconstructed cell layers. The total length and mean density were then calculated for each region. To calculate axonal and dendritic density distribution as a function of distance from soma or intrabulbar projection centroid, the total length of axons and dendrites within each doughnut-shaped bin were calculated using the Sholl Analysis function in Neurolucida Explorer, and this value was divided by the total area of the bin. To calculate directional distribution, the Wedge Analysis function was used. To estimate the area that a given neuron occupies in individual regions of the olfactory cortex, areas within a distance of $100 \mu \mathrm{m}$ from axons were computed ("axon filling rate"). A distance of $100 \mu \mathrm{m}$ was chosen based on the diameter of pyramidal cell dendritic extensions in the olfactory cortex (Johnson et al., 2000).

Cluster analysis. Morphometric parameters including total length, distance distributions, and direction distribution of both axons and dendrites for individual cells in the olfactory bulb were subjected to cluster analysis. For axons in the olfactory cortex, the mean axon density of individual cells across seven regions in the olfactory cortex was used to evaluate the strength of projections regardless of region size. Cluster analyses were then performed using Ward's algorithm.

Subareal comparison of MC/TC and TMT/2MBA projections. To compare the degree of MC and TC axonal projections in the olfactory cortex, the MC/TC score was mapped across the olfactory cortex space. The $\mathrm{MC} / \mathrm{TC}$ score was defined as $s=(\mathrm{ML}-\mathrm{TL}) /(\mathrm{ML}+\mathrm{TL})$, where $\mathrm{ML}$ is the total length of MC axons and TL is the total length of TC axons in individual $100 \times 100 \mu \mathrm{m}$ pixels. Thus, $s=1$ indicates that the projection was only from MCs, while $s=-1$ indicates that the projection was only from TCs. Values of MC/TC scores were represented on a color scale using three rounds of interpolation. For pixels without projection from either MCs or TCs, the color white was assigned, and thus the data from those pixels were not interpolated. To tentatively estimate the borders of these areas, pixels in which the $\mathrm{MC}$ and $\mathrm{TC}$ projections were nearly equal $(-0.2<s<+0.2)$ were extracted. Using these borders, the OC areas were divided into subareas, and the mean axon density was calculated. The axon density was then subjected to a statistical analysis to check the validity of the bordering procedure. These procedures are also used for TMT/2MBA score and comparison in TMT/2MBA projections.

Statistical analysis. To compare bin position, reliability index (see Fig. $2 D$ ), and axonal/dendritic length in the olfactory bulb (see Fig. 5C) and olfactory cortex (see Fig. 9D), a two-tailed unpaired $t$ test (a criterion of 
$p<0.05$ ) was used. Comparisons of axon length, axon density, and axon filling rate across multiple OC regions (see Fig. 8) were performed using ANOVA $(p<0.001)$ with a post hoc test (Tukey's HSD test, $p<0.05)$. In the analyses of concentration experiment (see Fig. 3D, 4C), post hoc comparisons between MCs and TCs (two-tailed unpaired $t$ test, a criterion of $p<0.05$ ) were made only if global analysis indicated a statistically significant difference between the cell types (ANOVA, $p<0.001$ ).

\section{Results}

Procedure for functional characterization and morphological visualization of individual MCs and TCs

To functionally identify individual MCs and TCs and visualize their axonal projections, an experimental procedure was developed consisting of the intrinsic optical imaging method, juxtacellular single-cell recording and labeling, and digital 3-D reconstruction techniques (Fig. 1A). Using the optical imaging of intrinsic signals (Rubin and Katz, 1999; Uchida et al., 2000), we first identified glomeruli that responded to the fox odor TMT in dorsal class II domain $\left(\mathrm{D}_{\mathrm{II}}\right)$ or to a spoiled food odor, $2 \mathrm{MBA}$, in dorsal class I domain $\left(\mathrm{D}_{\mathrm{I}}\right)$ (Kobayakawa et al., 2007; Matsumoto et al., 2010; Mori and Sakano, 2011). Because the two odorants represent functionally distinct domains $\left(D_{I}\right.$ and $\left.D_{I I}\right)$, projection neurons in the two domains may differ in their physiological response and axonal projection. Therefore, we first searched for the functional and connectivity differences between MCs and TCs, which were observed both for the TMTresponsive projection neurons and the $2 \mathrm{MBA}$-responsive projection neurons.

Figure $1 B$ shows an example of TMT-responsive glomeruli. We then penetrated a micropipette near the responsive glomeruli, and juxtacellularly recorded odor-induced spike responses of individual MCs or TCs. After the recording, an anterograde tracer was injected into the recorded cell using the juxtacellular electroporation method (Fig. 1D) (see Materials and Methods). To ensure that all labeled axons were derived from a single cell, only a single neuron was labeled in each OB. Brain sections containing the labeled neuron were then histochemically processed using an amplifying visualization technique and observed under a microscope (an MC in Fig. 1E). For visualization and quantitative analyses of axonal trajectory, dendritic and axonal arbors were digitally registered for 3 -D reconstructions (Fig. $1 F$ ). In the present study, TCs were further classified into eTCs, mTCs, and iTCs based on the position of their somata in the EPL according to the definition by Shepherd et al. (2004) (see Materials and Methods).

The OC consists of nine major areas: the AON, APC, olfactory tubercle, posterior piriform cortex (PPC), tenia tecta, nucleus of the lateral olfactory tract (nLOT), anterior cortical amygdaloid nucleus (ACO), posterolateral cortical amygdaloid nucleus (PLCO), and lateral entorhinal cortex (LEC) (Fig. $1 \mathrm{~F}$ ) (Heimer, 1968; Price, 1973). Figure $1 F$ shows an example of TMTresponsive $\mathrm{MC}$ axons projecting widely to all nine areas of the $\mathrm{OC}$ up to the LEC, which is the most distal area from the $\mathrm{OB}$ (Fig. $1 F$ ). Reconstructed TMT-responsive MCs $(n=7)$ had a longest axon of $15.3 \pm 1.1 \mathrm{~mm}$ (mean $\pm \mathrm{SE}$ ) with terminals in the LEC, a total of $273.2 \pm 30.4 \mathrm{~mm}$ of axons, and a total of $14.8 \pm 4.1 \mathrm{~mm}$ of dendrites. These results suggest that our procedure labels very long axon collaterals of the dye-injected neurons. Using the same procedure, we also recorded and labeled individual TMTresponsive TCs $(n=7)$, and then 3-D-reconstructed their axonal projections. TCs had shorter axons and dendrites than MCs: longest axon of $4.8 \pm 1.5 \mathrm{~mm}$ with terminals in the anterior OC areas, a total of $52.6 \pm 32.9 \mathrm{~mm}$ of axons, and a total of $5.8 \pm 2.9$ $\mathrm{mm}$ of dendrites ( $p<0.001$ or better for each comparison, $t$ test).
In pilot experiments without the amplifying axon-labeling procedure, distribution of en passant varicosities along the axon were assessed. The result showed that cumulative axon length correlated very well to a cumulative number of varicosities $(r=$ $0.991 ; p<10^{-15} ; n=785$ varicosities $)$. Together with the recent study reporting that $90 \%$ of $\mathrm{MC} / \mathrm{TC}$ axon termini have swelling in the olfactory cortex (Ghosh et al., 2011), the result suggests that axon length is a good indicator for estimating axon varicosities and terminal swellings. We observed no varicosities on the axon trunk running in the lateral olfactory tract, which is consistent with the previous study (Ghosh et al., 2011).

\section{TCs demonstrated shorter onset latency than MCs in response to odor inhalation}

Behavioral studies recently reported that rodents extract sufficient odor information within the first respiration of odor sampling (Uchida and Mainen, 2003; Abraham et al., 2004; Rinberg et al., 2006; Bathellier et al., 2008; Cury and Uchida, 2010). We thus focused our analysis to the timing of spike responses during the first respiration just after odor onsets (for $640 \mathrm{~ms}$ after the onset of odor inhalation, based on the result that a single respiration was $631 \pm 31 \mathrm{~ms}$ in our experimental condition; $n=10$ mice). Using single-cell recording of spike activity, temporal features of odor-induced spike activity were explored in individual TMT-responsive MCs and TCs ( $n=37 ; 17$ MCs and 20 TCs). Figure $2 A$ shows the reconstructed morphology of an eTC (TMTeTC), an mTC (TMT-mTC), and two MCs (TMT-MC). Their spike responses to odor stimulation were aligned at the onset of first odor inhalation after odor stimulation (Fig. 2B).

Because TCs and MCs typically demonstrated respiration phase-locked spontaneous spike activity even during blank stimulation (Fig. 2 B, see blank), the TMT-induced response should be assessed by comparing the activity during blank stimulation with the TMT-induced spike activity (Fig. $2 B$, red and blue traces). As an index to assess how much information individual cells encode in odor response compared with spontaneous firing, we defined a metric, reliability index (RI), using ROC analysis, a method based on signal detection theory (see Materials and Methods) (Feierstein et al., 2006; Cury and Uchida, 2010). RI indicates the reliability of the spike response of a neuron to a particular stimulus compared with spontaneous activities. Figure $2 C$ shows plots of RI as a function of time after the onset of first odor inhalation. For the ROC analysis, we examined six different temporal bin sizes (10-320 ms; from blue to red in Fig. $2 C$ ).

To determine whether MCs and TCs have different onset latency in encoding reliable responses, we identified statistically significant RI points that emerged earliest ( $\mathrm{RI}_{\mathrm{ES}}$ ) (Fig. $2 \mathrm{C}$, arrows). Comparison of $\mathrm{RI}_{\mathrm{ES}}$ between cell types in the population revealed that TCs have considerably shorter latency in expressing reliable responses than MCs (Fig. 2D; TC, $108 \pm 12 \mathrm{~ms}$; MC, $252 \pm 12 \mathrm{~ms} ; p<0.00001)$. Bin sizes that expressed $\mathrm{RI}_{\mathrm{ES}}$ in TCs were smaller than those for MCs (TC, $20.8 \pm 1.2 \mathrm{~ms}$; MC, $52.2 \pm$ $1.3 \mathrm{~ms} ; p<0.01$ ), suggesting that TCs communicate with shorter temporal features. However, the degree of reliability achieved with these onsets did not differ between cell types (Fig. $2 D$, right; TC, $0.83 \pm 0.03 ; \mathrm{MC}, 0.79 \pm 0.03 ; p=0.23)$.

\section{TCs maintained shorter latency spike responses with a lower concentration threshold than MCs}

We next examined whether TCs also show shorter onset latency at lower odor concentration. Cells may conceivably need a larger temporal window to accumulate enough information with weak sensory input, and thus a difference between TCs and MCs may 
A Intrinsic Signals ......(B)
Juxtacellular
Single-cell Recording ...(C)
Jingle-cell Labeling ...(D)
3-day Survival for
Tracer Transport
Perfusion/Tracer
Visualization .....(E)
3-D Reconstruction ....(F)
B

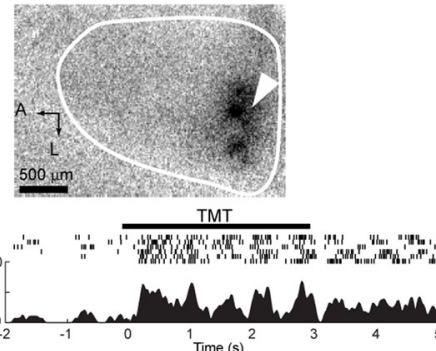

D

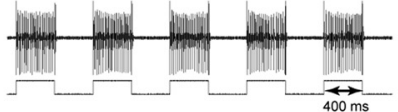

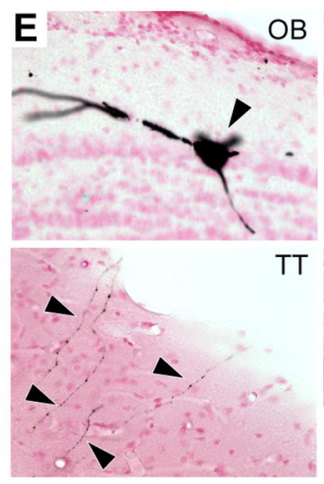
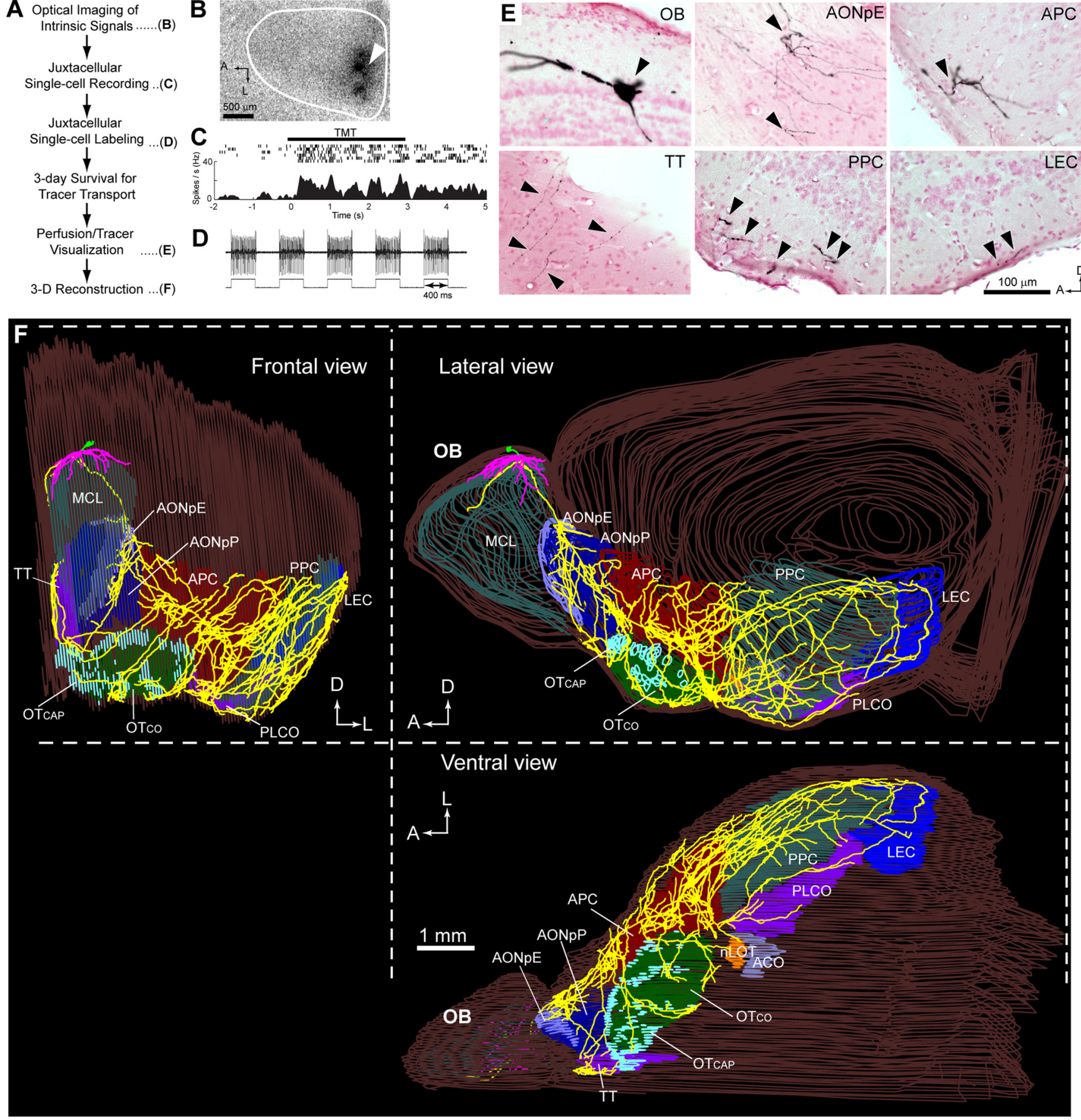

Figure 1. Functional characterization and morphological visualization of mitral and tufted cells. $\boldsymbol{A}$, The sequence of optical imaging, juxtacellular recording, and labeling methods for the visualization of a functionally identified neuron. $\boldsymbol{B}$, Optical imaging of intrinsic signals showing TMT-responsive glomeruli (dark spots) in the exposed dorsal surface (white line) of the left OB. A recording micropipette was lowered near the glomerulus (arrowhead). A, Anterior; L, lateral. C, Juxtacellular single-cell recording from a TMT-responsive MC. Spike responses to TMT odor are shown with raster representation (top traces) and a peristimulus time histogram (bottom graph). $\boldsymbol{D}$, Juxtacellular single-cell electroporation. Current-induced firing of the neuron (top trace) is perfectly locked to the positive current pulses ( $400 \mathrm{~ms}$ on/off; bottom trace) applied to the recorded neuron, indicating successful electroporation. $\boldsymbol{E}$, Visualized cell body and dendrites of the labeled MC in the OB, a dense axon collateral bush in the pars externa of the anterior olfactory nucleus (AONpE), an axon collateral bush in the anterior piriform cortex (APC), and axon collaterals in the tenia tecta (TT), posterior piriform cortex (PPC), and lateral entorhinal cortex (LEC). Axons were clearly visualized in all OC regions. $\boldsymbol{F}$, Three-dimensional reconstruction of a single TMT-responsive MC. The left hemisphere of the brain containing the labeled cell is shown for frontal (left), lateral (top right), and ventral (bottom right) views. Axons, primary dendrites, and lateral dendrites are indicated by yellow, green, and magenta, respectively. The mitral cell layer (MCL) of the $\mathrm{OB}$ and different areas of the $\mathrm{OC}$ are indicated by distinct colors and superimposed on the line tracing of the brain surface (brown). Abbreviations: $\mathrm{AONpP}$, Pars principalis of anterior olfactory nucleus; $0 \mathrm{~T}$, olfactory tubercle; $0 \mathrm{~T}_{\text {CAP, }}$ cap part of olfactory tubercle; $0 \mathrm{~T}_{\mathrm{CO}}$, cortical part of olfactory tubercle; $\mathrm{nL} \mathrm{OT}$, nucleus of the lateral olfactory tract; $\mathrm{ACO}$, anterior cortical amygdaloid nucleus; $\mathrm{PLCO}$, posterolateral cortical amygdaloid nucleus; $\mathrm{A}$, anterior; $\mathrm{D}$, dorsal; L, lateral.

become smaller at low odor concentrations. We therefore assessed cellular latencies using a decreasing concentration series of TMT $(n=15$ cells; $n=7$ MCs and $n=8$ TCs; measured with all concentrations). Examination of the odor concentration-re- sponse relationship revealed the stimulus threshold for activating TCs to be at low concentrations ranging from 1.5 to $7.1 \mathrm{ppb}$ (Fig. $3 A$, mTC). By contrast, the stimulus threshold was $\sim 10-100$ times higher in MCs (Fig. 3A, MC). At higher concentrations of 
A
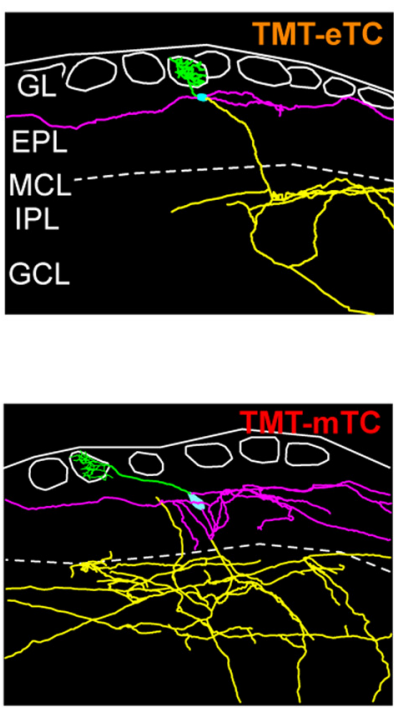

B
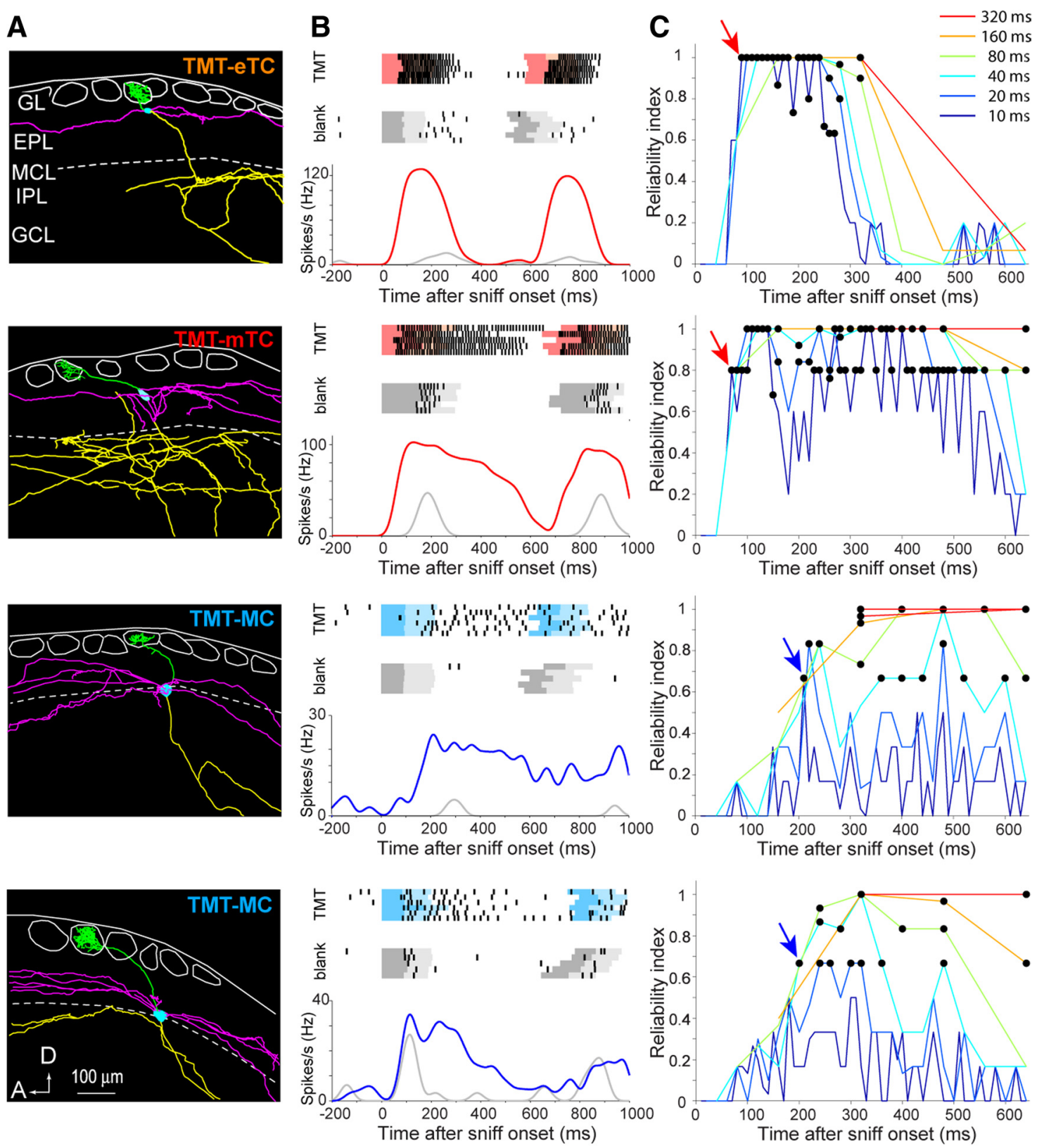

D

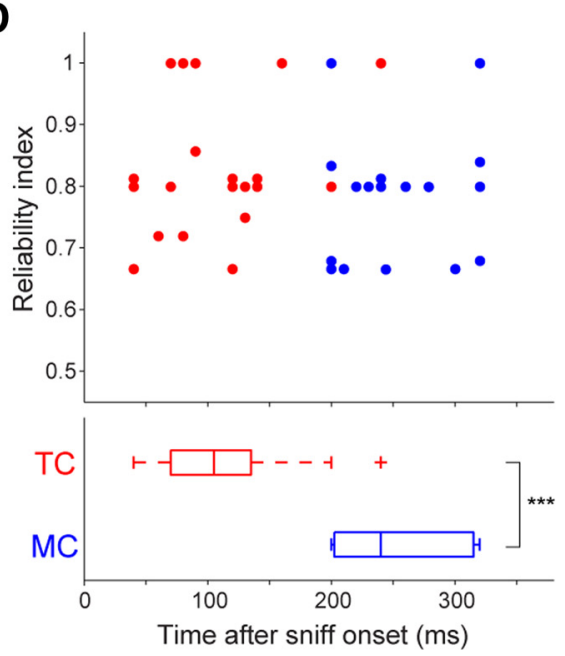

Figure 2. Signal timing was faster in tufted cells than mitral cells. A, Morphological reconstruction of an eTC, an mTC, and two MCs (from top to bottom) that responded to fox odor TMT. Cell bodies, axons, primary dendrites, and lateral dendrites are indicated by blue, yellow, green, and magenta, respectively. These color codes are used in all subsequent figures. (Figure legend continues.) 
A
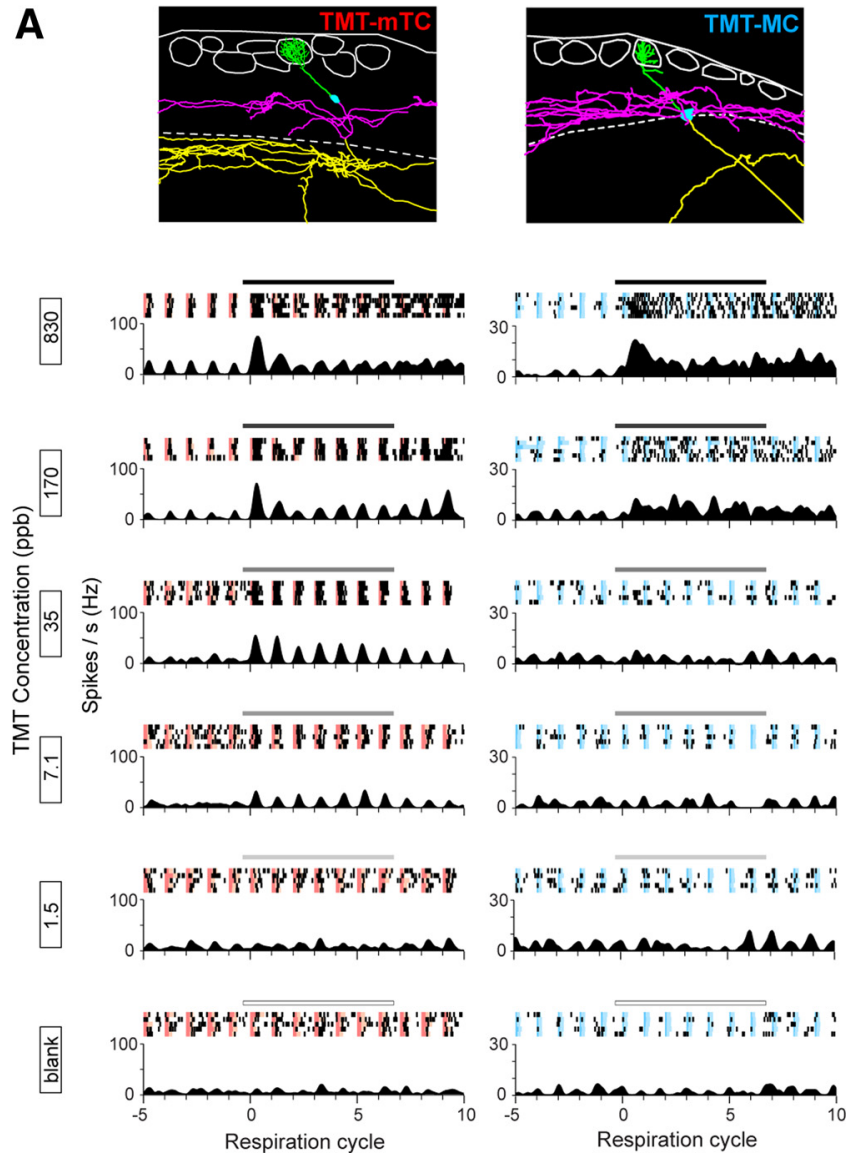

B
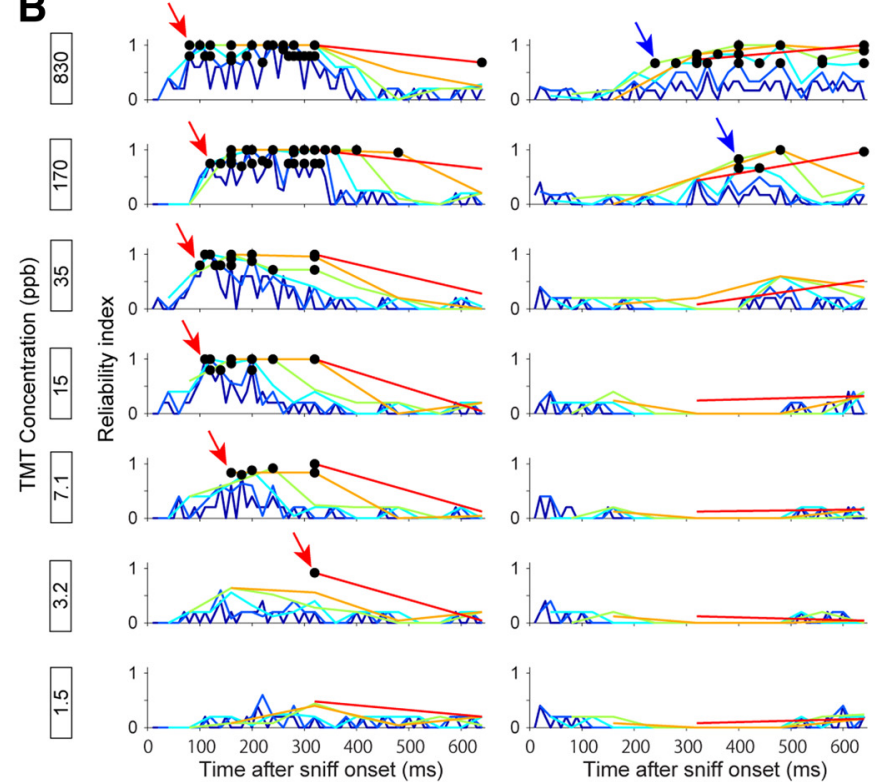

C
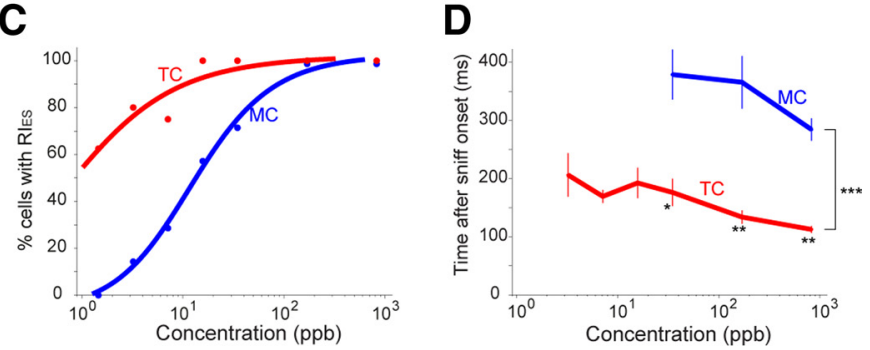

Figure 3. Short-onset latencies of tufted cells were maintained over a wide range of odor concentration series. A, Top, Morphological reconstruction of a middle TC (TMT-mTC) (left) and MC (TMT-MC) (right) that responded to TMT. Layer structure is shown as in Figure 2A. Bottom, Raster plots of spike discharges and peristimulus-phase histograms of the mTC and MC in response to stimulation (black bar, $4 \mathrm{~s}$ ) with different concentrations of TMT ( $830-1.5 \mathrm{ppb}$, from top to bottom). $\boldsymbol{B}$, Plot of Rl of the mTC (left) and MC (right) shown in $A$ in response to TMT concentration series (830-1.5 ppb, from top to bottom). Rl was plotted using six different temporal bins as in Figure 2. C, Proportion of MCs (blue; $n=7)$ and TCs (red; $n=8)$ that exhibited statistically significant RI in odor concentration series. The lines indicate logistic fitting of the plots. $\boldsymbol{D}$, Bin position that exhibited the earliest significant RI ( $\left(\mathrm{RI}_{\mathrm{ES}}\right)$ in MC (blue; $\left.n=7\right)$ and TC (red; $\left.n=8\right)$ populations in the TMT concentration series. $\mathrm{RI}_{\mathrm{ES}}$ emerged faster in TCs than MCs at all concentrations. Note that only concentrations at which more than two-thirds of MCs demonstrated RI $\mathrm{ES}_{\mathrm{ES}}$ are plotted, and most MCs did not demonstrate $\mathrm{RI}_{\mathrm{ES}}$ at low concentrations. ${ }^{*} p<0.01$ ( $t$ test), ${ }^{* *} p<0.001$ ( $t$ test), ${ }^{* *} p<1.0 \mathrm{e}-12$ (ANOVA).

TMT, both MCs and TCs demonstrated robust spike responses. Figure $3 B$ shows how the reliability of $\mathrm{mTC}$ and $\mathrm{MC}$ responses depends on TMT concentration. Surprisingly, the mTC exhibited the earliest significant $\mathrm{RI}\left(\mathrm{RI}_{\mathrm{ES}}\right)$ down to very low concentration (3.2 ppb), whereas the MC exhibited significant RI only at high concentrations (830 and $170 \mathrm{ppb}$ ). In the population, ap-

$\leftarrow$

(Figure legend continued.) Layer structure is shown as follows: GL, glomerular layer; EPL, external plexiform layer; $M C L$, mitral cell layer; IPL, internal plexiform layer; $G C L$, granule cell layer. The white circles in the $\mathrm{GL}$ indicate glomeruli. A, Anterior; D, dorsal. B, Timing of spike responses of the four cells shown in $A$. Top and middle, Raster plots of spike trains evoked by the inhalation of $830 \mathrm{ppb}$ TMT (red or blue; top plots) or by blank air (gray; middle plots). Each row corresponds to a single trial. The darker and lighter colored shadings behind rasters indicate the respiration inhalation and exhalation, respectively. Bottom, Peristimulus time histogram of spike response to TMT stimulation (red or blue) and blank (gray). In all analyses, time 0 is shown by aligning at the first onset of odor inhalation. C, Plots of response reliability index of the cells in $A$ as a function of time. Rl was plotted using six different bin lengths ( $10-320 \mathrm{~ms}$; blue to red). The black circles indicate a statistically significant RI value of the response of each cell (see Materials and Methods). The arrows indicate earliest significant $R I\left(R I_{E S}\right)$. D, Top, Plot of $\mathrm{RI}_{E S}$ for individual cells $(n=37)$. Each point indicates a single MC (blue; $n=17)$ or TC (red; $n=20)$. The horizontal axis indicates the bin position at which $\mathrm{Rl}_{\mathrm{ES}}$ emerged, and the vertical axis indicates the $\mathrm{RI}$ value when $\mathrm{RI}_{\mathrm{ES}}$ emerged. Bottom, Box plot showing the median bin position of $\mathrm{RI}_{\mathrm{ES}}$ for MCs and that for TCs. Right, Box plot showing the RI value of RI for MCs and TCS. ${ }^{* *} p<$ 0.00001 . proximately one-half of recorded TCs exhibited $\mathrm{RI}_{\mathrm{ES}}$ at low concentration (1.4 ppb), whereas one-half of MCs expressed $\mathrm{RI}_{\mathrm{ES}}$ only above medium concentration ( $\sim 15$ ppb; Fig. $3 C$ ), indicating that TCs have a much wider dynamic range for odor concentration than MCs. As predicted, onset latencies of both MCs and TCs became larger as concentration decreased, suggesting that encoding significant reliable responses requires a longer temporal window at low concentration (Fig. 3D). Nevertheless, at all odor concentrations, TC latencies were significantly shorter than MC latencies (Fig. $3 D ; p<0.01$ or better). Comparison between 2MBA-responsive MCs and TCs in dorsal class I domain also indicated shorter latencies in TCs in all concentrations (Fig. 4). These results suggest that TCs convey fast signals with shorter latency than MCs at a wide range of odor concentration.

We examined spatial distribution of tangential distance between recorded TMT-responsive MC/TC somas and their glomeruli for cells that could be fully reconstructed ( $n=20 \mathrm{MCs}$ and $n=15$ TCs, respectively). The result showed that the tangential distances between glomerulus and soma were not significantly different between sampled MCs and TCs $(p=0.32, t$ test). The recorded TMT-responsive MC somas were distributed at distances $115 \pm 62$ and $190 \pm 132 \mu \mathrm{m}$ tangentially in the medial and posterior direction from the glomerulus, respectively, while TMT-responsive TC somas were distributed at distances $70 \pm 57$ 
A

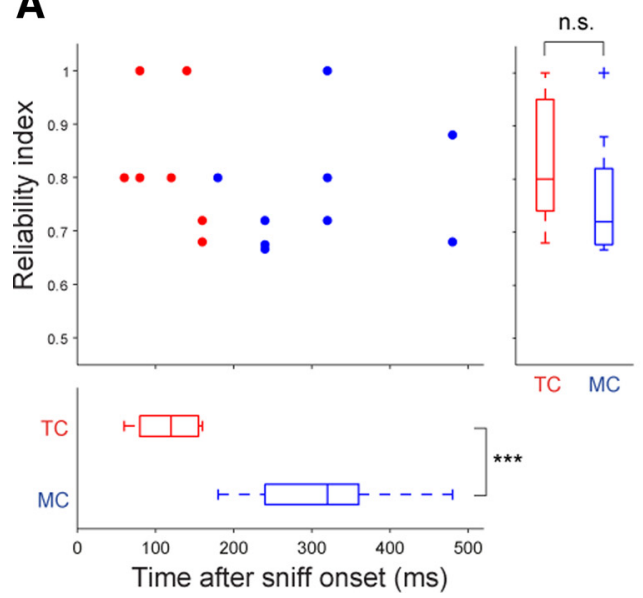

B

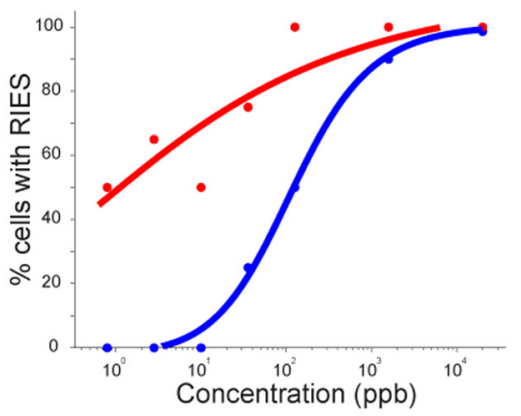

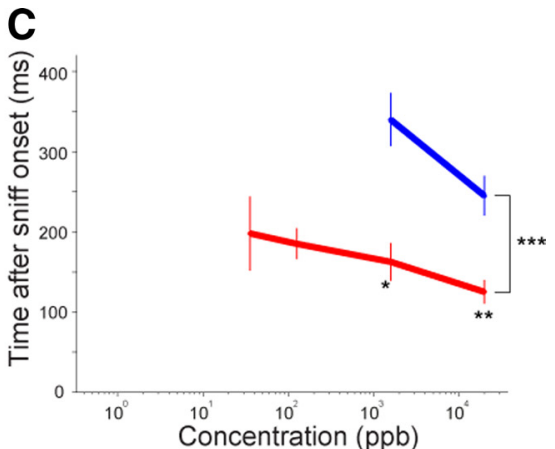

Figure 4. Signal timing in 2MBA response was faster in tufted cells than mitral cells. $A$, The same analysis as in Figure 2D for 2MBA-responsive MCs and TCs in $D_{1}$ domain ( $n=16 ; n=9$ MCs and $n=7 \mathrm{TCs} ; 20.0 \mathrm{ppm} 2 \mathrm{MBA}$ ). 2MBA-responsive TCs also had considerably shorter latency in expressing reliable responses than MCs (TC, $114 \pm 16 \mathrm{~ms}$; MC, $313 \pm 35 \mathrm{~ms}$; $\left.{ }^{* * *} p<0.001\right)$. $\boldsymbol{B}, \boldsymbol{C}$, Same analyses as in Figure 3, C and D, respectively, for 2MBA-responsive MCs and TCs using concentration series ( $n=8 ; n=4 \mathrm{MCs}$ and $n=4 \mathrm{TCs}$ ). 2 MBA-responsive TCs maintained short-latency spike responses with a lower concentration threshold than $2 \mathrm{MBA}$-responsive MCs. ${ }^{*} p<0.05,{ }^{* *} p<0.01,{ }^{* * *} p<0.001$.

and $164 \pm 125 \mu \mathrm{m}$ tangentially in the medial and caudal direction, respectively. Furthermore, we found no correlation between onset latencies and soma-glomerulus distance $(n=35$ cells; $r=$ $0.21, p=0.22$ ). Thus, the highly overlapping spatial distribution of MC and TC somas suggests that the clear distinction of response onset latencies and odor concentration threshold does not result from the sampling bias to record from TCs that are close to and associate with the strongly activated glomeruli and to record from MCs that are distant from the glomeruli and associate with weakly activated glomeruli.

\section{Dendritic and axonal projection of MCs and TCs within the olfactory bulb}

In addition to the difference in the projection of lateral dendrites, TCs differ from MCs in having extensive recurrent axons in the internal plexiform layer (IPL) and mirror-symmetric intrabulbar projections (Schoenfeld et al., 1985). However, which cell subtypes form these recurrent projections remains controversial (Belluscio et al., 2002; Lodovichi et al., 2003; Ghosh et al., 2011). We thus analyzed the local dendritic and axonal projections of recorded MCs and TCs within the OB. Figure $5 \mathrm{~A}$ shows $3-\mathrm{D}$ representations of dendritic and axonal projections of TMTresponsive eTC (left), mTC (center), and MC (right). Lateral dendrites of the labeled neurons covered the posterior part of the dorsal surface of the OB. Lateral dendrites of TCs were distributed preferentially in the superficial half of the EPL, while those of MCs were primarily present in the deeper half of the EPL, in agreement with previous studies (Macrides and Schneider, 1982; Mori et al., 1983).

By visual inspection, the extent of lateral dendrite projection was largest in MC, intermediate in $\mathrm{mTC}$, and smallest in eTC. By contrast, local axon collaterals of the eTC and mTC were prominent within the OB. Both the eTC and mTC had several intrabulbar projection axons that projected to the mirror-symmetric position (Fig. $5 A$, arrowheads) in the medial part of the $\mathrm{OB}$ to form a dense bush of axon terminals as well as recurrent axon terminals in the vicinity of the cell body (Fig. $5 A$, arrows). We visualized nine MCs and eight TCs in total within the $O B$, and these distinctions were conserved for all examined MCs and TCs.

To visualize the population distribution of dendrites and axons of labeled MCs or TCs, we constructed two-dimensional den- sity maps of dendrites and axons for TMT-responsive TCs (Fig. $5 B$, top maps) and MCs (Fig. 5B, bottom maps). Quantitative analysis confirmed that MCs had larger and denser dendritic fields than TCs (Fig. 5B,C). As for axons, however, TCs had considerably more extensive local collaterals than MCs. In the axon density maps, dense local axon collaterals beneath the cell bodies (an arrow) and the intrabulbar projection axons that targeted the mirror-symmetric position in the medial map (an arrowhead) are depicted as double peaks (Fig. 5B). Although all reconstructed TCs including eTC, $\mathrm{mTC}$, and iTC had intrabulbar projections, no MCs had them.

Cluster analysis was performed to quantitatively confirm the distinct projection patterns of lateral dendrites and local axon collaterals between MCs and TCs (Kozloski et al., 2001; Wong et al., 2002). Morphometric parameters of dendrites and axons including total length (Fig. 5C), distance distributions, and direction distributions were subjected to cluster analysis for individual cells (see Materials and Methods). The analysis revealed that the 17 cells were clustered into two major groups that coincided with TC and MC groups (Fig. 5D). The linkage distance was $9800 \pm$ 660 for any two TCs and $6400 \pm 370$ for any two MCs. Compared with the linkage distances within each group, the linkage distance between an MC and any TC was significantly larger $(18,000 \pm$ 460) $\left(p<1.0 \times 10^{-15}\right)$. In summary, MCs and TCs show distinct projection patterns of lateral dendrites and local axon collaterals within the OB. All TC subtypes had dense axonal projections both beneath the cell body and at the mirror-symmetric sites of the medial part.

\section{Axonal projection patterns of MCs and TCs in the olfactory cortex}

Next, we analyzed the axonal projection patterns of individual MCs and TCs in the OC. To represent 3-D reconstructions of axons in a single figure, the ventrolateral view of the reconstruction was used (Figs. 6, 7). Axons in the tenia tecta, which is hidden in the medial wall of the brain, are shown in a separate medial view (Fig. 6, bottom left insets).

Figure $6 \mathrm{~A}$ shows the axonal projection of an example TMTresponsive $\mathrm{MC}$. This cell extended a single main axon that ran through the lateral olfactory tract at the surface of the AON and APC. As was the case for the MC in Figure $1 F$, the main axon of 
A

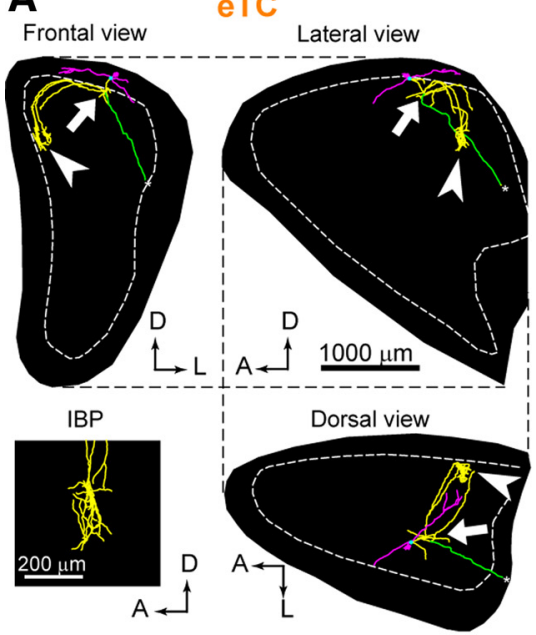

B

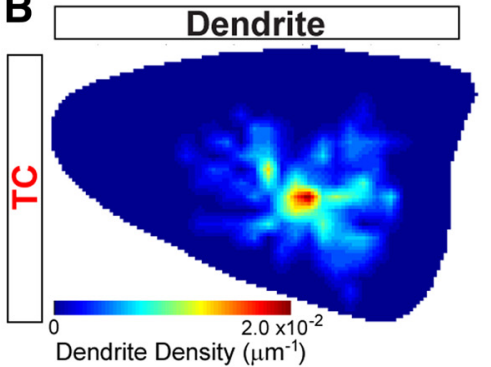

Dendrite Density $\left(\mu \mathrm{m}^{-1}\right)$

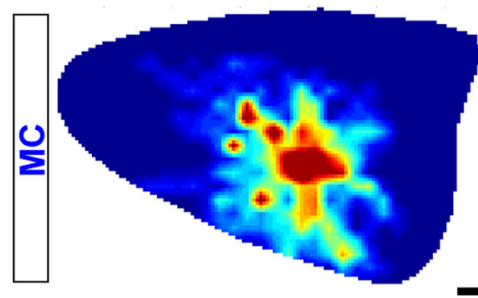

mTC

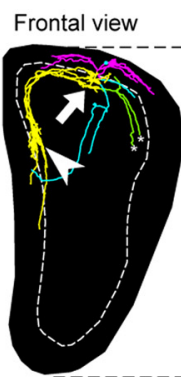

IBP

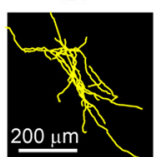

Lateral view

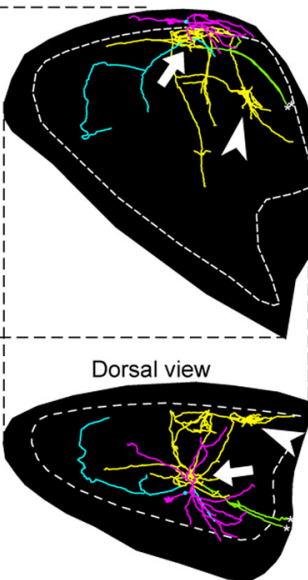

C

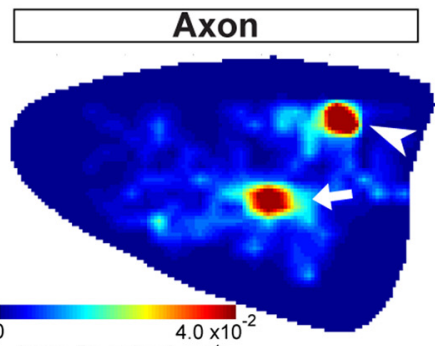

Axon Density $\left(\mu \mathrm{m}^{-1}\right)$

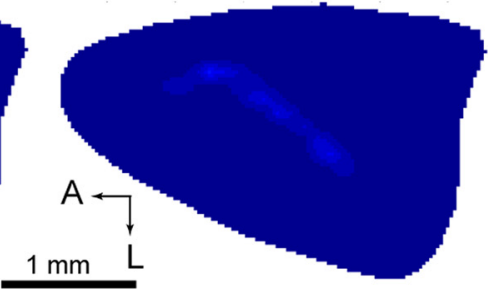

D
MC
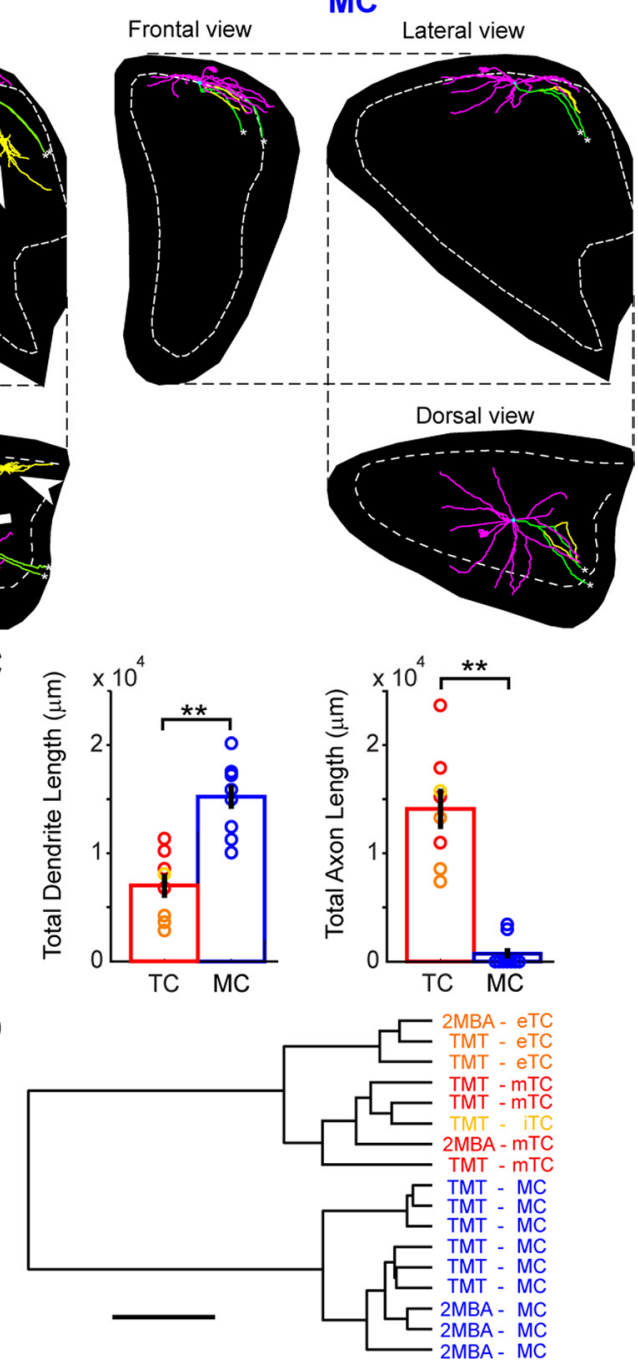

Figure 5. Tufted cells have extensive local axonal projections within the olfactory bulb. $\boldsymbol{A}$, Three-dimensional reconstruction of axons and dendrites in the $0 \mathrm{~B}$ for TMT-responsive eTC, mTC, and MC. Reconstructed cells are shown for frontal (top left), lateral (top right), and dorsal (bottom right) views of the OB. Intrabulbar projection (IBP) axon bushes (arrowhead) of TCs are also shown in a magnified lateral view (left bottom). Axons running to the $O C$ and to the anterior $0 B$ portion are shown in green and blue, respectively. The black backgrounds indicate projected surfaces of the $0 B$. The dotted line shows the mitral cell layer at its largest size. A, Anterior; D, dorsal; L, lateral. $B$, Dendrite and axon density maps for TMT-responsive TCs ( $n=8$; top) and TMT-responsive MCs ( $n=$ 9; bottom). Note that the TCs have a peak in axon density at the IBP (arrowhead) in addition to the near-soma area (arrow). $C$, Total dendrite (left) and axon (right) length for TCs ( $n=8$; red) and MCs ( $n=9$; blue) within the OB. ${ }^{* *} p<0.001, t$ test. $D$, Cluster analysis of dendritic and axonal projection patterns of individual MCs and TCs. The hierarchical tree grouped 17 neurons into two clusters according to their similarity in dendritic and axonal projection patterns within the $0 \mathrm{~B}$. These two clusters coincide with $\mathrm{MC}$ and TC groups. The horizontal bar indicates $1.0 \times 10^{4} \mathrm{U}$ in Euclidean distance.

this cell emitted many collaterals that projected extensively to all nine OC regions. In the most anterior part of the OC, axon collaterals of the MC formed a dense cluster at the pars externa of the $\mathrm{AON}$ (AONpE). The main axon of the MC also emitted collaterals that projected to the anterolateral and dorsal parts of the AON pars principalis $(\mathrm{AONpP})$ but did not reach the posteroventral and medial parts. Some axons in the AONpP extended ventrally and reached the anterior half of the tenia tecta.

In the ventral part of the APC, several clusters of high-density axon collaterals were distributed, which is consistent with our previous findings in rabbits (Ojima et al., 1984). Some axons that branched ventrally in the APC extended to the medial and posterior parts of the olfactory tubercle. From the posterior part of the APC, many axon collaterals ran posteriorly for long distances through the PPC, and some of these reached the LEC. The axon collaterals of the MC covered a large portion of the PPC. The axons also projected to the nucleus of the lateral olfactory tract, anterior cortical amygdaloid nucleus, and posterolateral cortical amygdaloid nucleus. We reconstructed the axonal projections of seven MCs, and all of these MCs showed the characteristic axon collateral projection that reached every area of the OC (four TMT- and three 2MBA-responsive MCs; Figs. 6, 7).

By contrast, the axons of TMT-responsive mTCs (Fig. 6D) demonstrated completely different projection patterns from those of TMT-responsive MCs. The axons of the TMTresponsive $\mathrm{mTC}$ branched in the $\mathrm{OB}$ and projected to the anterior areas of the $\mathrm{OC}$ ( $\mathrm{AON}, \mathrm{APC}$, and olfactory tubercle). However, the axons did not reach the more posterior areas of the OC, in agreement with previous anatomical and electrophysiological studies (Haberly and Price, 1977; Scott, 1981; Luskin and Price, 1982). Axon collaterals of the mTC formed high-density clusters in the AONpE and the posteroventral part of the AONpP. The axons projected further to the border of the APC and olfactory tubercle, covering mainly the "cap" part of the olfactory 

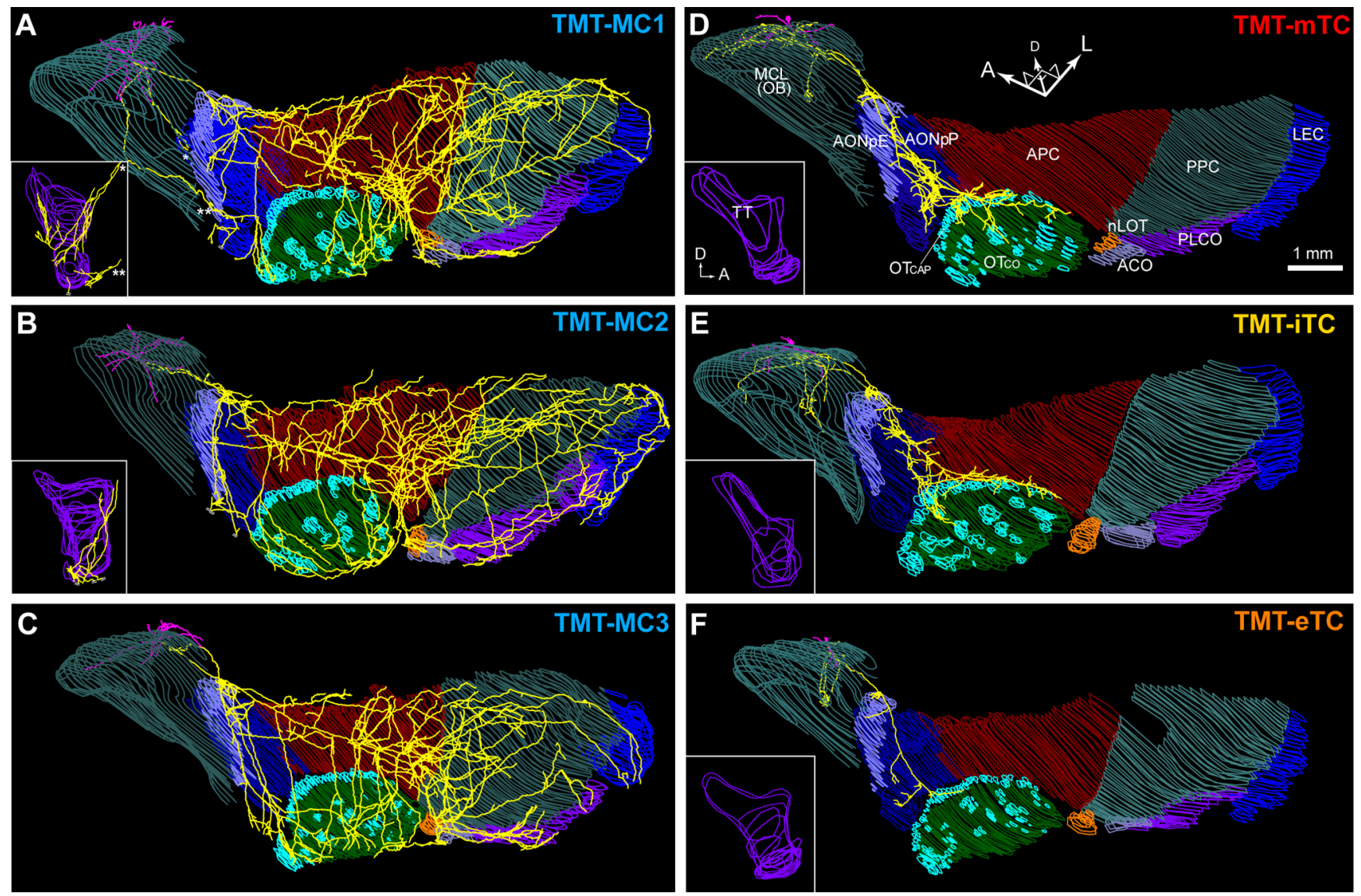

Figure 6. Axonal projection of TMT-responsive mitral and tufted cells in the olfactory cortex. $A-C$, Axonal projection of three TMT-responsive MCs in the $0 C$. The 3-D brain reconstruction containing the cell was rotated to a ventrolateral view. Dendrites (magenta) and axons (yellow) are shown with the mitral cell layer of the $O B$ and layer $I l$ of each region of the $O C$. Inset, The medial view of axons in the tenia tecta, which is hidden in the ventrolateral view. Note that the tenia tecta of the MC in C could not be reconstructed because of section damages. $\boldsymbol{D}-\boldsymbol{F}$, Axonal projection of a TMT-responsive $\mathrm{mTC}(\boldsymbol{D})$, iTC $(\boldsymbol{E})$, and eTC $(\boldsymbol{F})$ to the $0 C$. Abbreviations: $\mathrm{ACO}$, Anterior cortical amygdaloid nucleus; $\mathrm{AONpE}$, pars externa of the anterior olfactory nucleus; $A 0 \mathrm{NpP}$, pars principalis of anterior olfactory nucleus; $\mathrm{APC}$, anterior piriform cortex; $\mathrm{LEC}$, lateral entorhinal cortex; $\mathrm{MCL}$, mitral cell layer; $0 \mathrm{~T}$, olfactory tubercle; $0 \mathrm{~T}_{\mathrm{CAP}}$, cap part of olfactory tubercle; $0 \mathrm{~T}_{\mathrm{CO}}$, cortical part of olfactory tubercle; nLOT, nucleus of the lateral olfactory tract; PLC0, posterolateral cortical amygdaloid nucleus; PPC, posterior piriform cortex; TT, tenia tecta; A, anterior; D, dorsal; L, lateral.

tubercle (Meyer and Wahle, 1986) and the medial edge of the ventrorostral part of the APC. No axon was observed in the other regions of the OC. We reconstructed seven TCs, and all these TCs had their axonal projection limited to the anterior areas of the OC (four TMT- and three 2MBA-responsive TCs; Figs. 6, 7).

To quantitatively assess the axon projection patterns of MCs and TCs in the OC areas, three parameters assessing axon projection patterns were computed across different cortical areas (Fig. $8 A-C$ ), and population axon density maps were constructed (Fig. $9 A, B)$. In both TMT- and 2MBA-responsive MCs, these analyses indicated three characteristic projection patterns: (1) MC axons projected to almost every area of the $\mathrm{OC}$, although axon collateral density varied among target areas (Fig. 8B); and (2) MCs formed several high-density clusters of axon collaterals along the ventromedial part of the APC (Fig. 9A, red scattered spots), while they projected axon collaterals sparsely over most part of the APC and PPC (Fig. 8C). Thus, axons of individual MCs showed a biased, and at the same time highly distributed, projection pattern in the $\mathrm{APC}$ and PPC.

By contrast, axon density maps of TMT- and 2MBAresponsive TCs indicated the following four characteristics: (1) axons of TCs projected only to the anterior areas of the OC (AON, APC, and olfactory tubercle); (2) TCs formed a small cluster of axon collaterals at a specific position of the AONpE and some clusters in the posteroventral part of the AONpP; (3) TCs formed clusters of terminals along the border between the cap region of the olfactory tubercle and the medial edge of the APC; and (4) TCs did not project axons to the medial and posterior areas of the OC. Among TCs, eTCs tended to project fewer axon collaterals in the anterior areas (Figs. $6 F, 7 F$ ), but all of the TC subtypes projected axons selectively within the AON, APC, and olfactory tubercle.

In a cluster analysis of MCs and TCs based on their axon densities across the individual OC areas (Fig. 8 B), a total of 14 neurons was clustered into two major groups that coincided again with the TC and MC groups (Fig. $8 D$ ). These results confirmed that MCs and TCs show a distinct pattern of axon projections in the OC.

MCs and TCs project axons to segregated subareas in the OC Finally, we asked, with respect to the anterior OC areas, whether the MCs and TCs projected to segregated or shared target sites within each area. Averaged axon density map of the MCs (Fig. 9A) was compared with that of the TCs (Fig. 9B). Subtraction of the averaged axon density map of TCs from that of MCs (Fig. 9C) clearly indicated the difference in the subarea dominated by TC axons and the subarea dominated by $\mathrm{MC}$ axons in each of the anterior areas (AONpE, AONpP, APC, and olfactory tubercle) of the OC.

To tentatively define borders of these subareas, pixels where the MC and TC projections were nearly equal were extracted (Fig. 

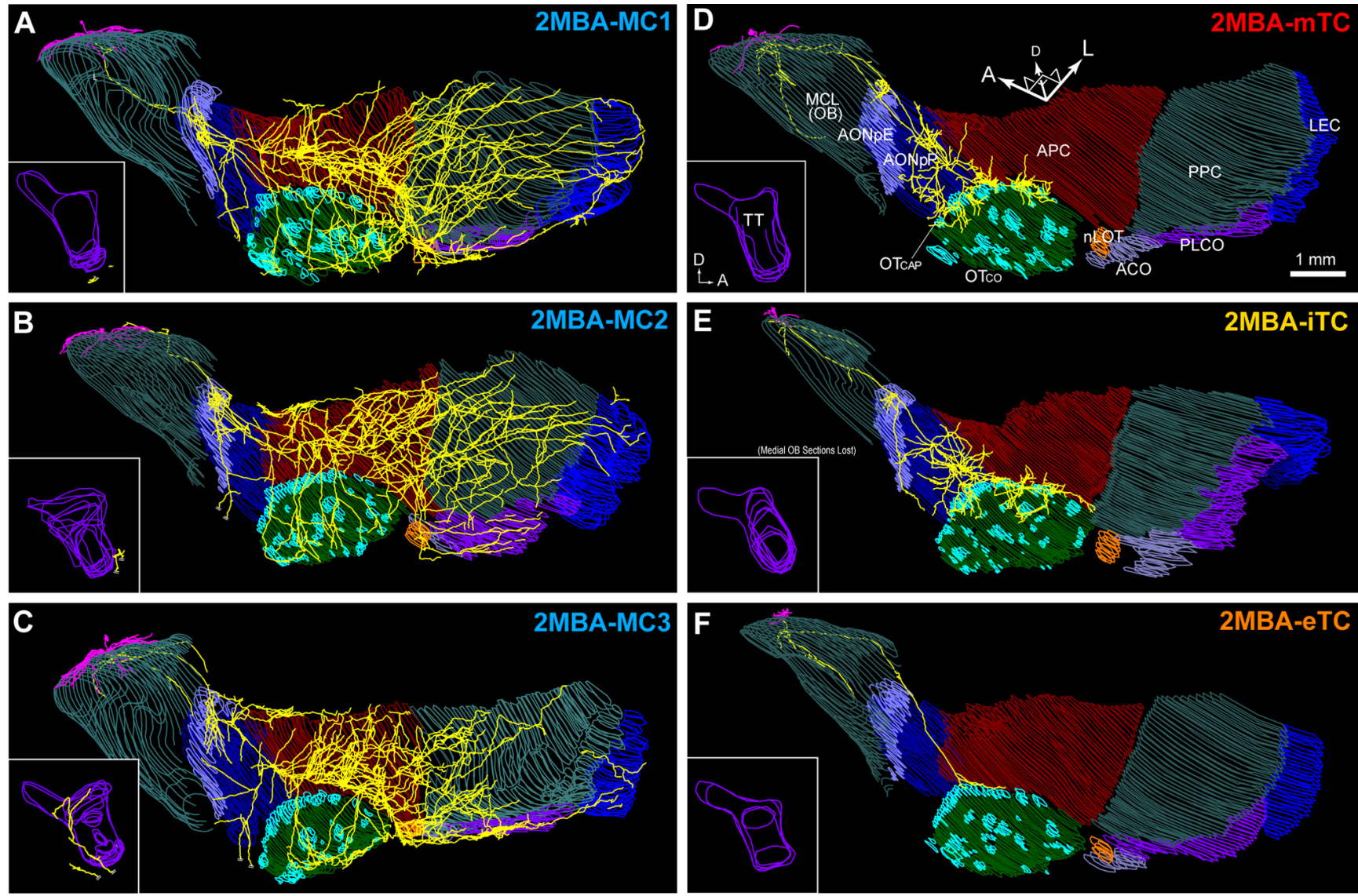

Figure 7. Axonal projection of 2MBA-responsive mitral and tufted cells in the olfactory cortex. $A-C$, Axonal projection of three 2MBA-responsive MCs in the $0 C$ in ventrolateral view. Dendrites (magenta) and axons (yellow) are shown with the layer Il of each region of the 0 C. Inset, A medial view of the axons in the tenia tecta (TT). D-F, Axonal projection of a 2 MBA-responsive mTC (D), iTC $(\boldsymbol{E})$, and eTC $(\boldsymbol{F})$ to the $O C$. Abbreviations: ACO, Anterior cortical amygdaloid nucleus; $A 0 \mathrm{NpE}$, pars externa of the anterior olfactory nucleus; AONpP, pars principalis of anterior olfactory nucleus; $\mathrm{APC}$, anterior piriform cortex; $\mathrm{LEC}$, lateral entorhinal cortex; $\mathrm{MCL}$, mitral cell layer; $\mathrm{OB}$, olfactory bulb; $0 \mathrm{~T}_{\mathrm{CAP}}$, cap part of olfactory tubercle; $0 \mathrm{~T}_{\mathrm{CO}}$, cortical part of olfactory tubercle; $\mathrm{nL} \mathrm{OT}$, nucleus of lateral olfactory tract; PLCO, posterolateral cortical amygdaloid nucleus; PPC, posterior piriform cortex; A, anterior; D, dorsal; L, lateral.

$9 A-C$, broken line) (see Materials and Methods). Using these borders, the AON, APC, and olfactory tubercle were divided into seven subareas (Fig. 9C, 1-7). In the AON, clusters of TC axon collaterals were largely confined to the anterior-most dorsolateral corner (subarea 1) and to the triangle-shaped area at the posteroventral part (subarea 3), while those of MCs were distributed in the anterolateral and dorsal parts (subarea 2). In the olfactory tubercle, TC axons projected selectively to the anterolateral edge (mainly terminating in the cap region, subarea 5), whereas MC axons projected widely, covering the medial and posterior parts (subarea 4). In the APC, TC axons projected only to the medial edge of the ventrorostral part (subarea 7), while MC axons tended to avoid this part and projected widely to the rest of the APC (subarea 6). Statistical comparison confirmed the above distinctions within these seven subareas (Fig. 9D; $p<0.01$ ). These results indicate that MCs and TCs project to segregated subareas within each of the anterior OC areas.

Since we labeled TMT- and 2MBA-responsive MCs and TCs in $D_{I}$ and $D_{I I}$, respectively, it is of interest to ask whether the cells in these different $\mathrm{OB}$ domains have different projection patterns in the OC. We thus divided our samples into TMT-MC/TCs group ( $n=8$; including $n=4$ TMT-MCs and $n=4$ TMT-TCs) and 2MBA-MC/TCs group $(n=6$; including $n=32 \mathrm{MBA}$-TCs and $n=32 \mathrm{MBA}-\mathrm{TCs}$ ), and compared axonal projections (Fig. $9 E-G)$. The results showed that TMT-MC/TCs had more axonal projection in the tenia tecta and posterodorsal part of the
$\mathrm{AON}$, whereas $2 \mathrm{MBA}-\mathrm{MC} / \mathrm{TCs}$ had more projection in the ventral half of the APC and the middle part of the olfactory tubercle (Fig. 9H). These results indicate that some of the OC areas preferentially receive input from either TMT-MC/TCs or 2MBA-MC/TCs.

\section{Discussion}

The present study demonstrates fundamental distinctions between TMT/2MBA-responsive MCs and TCs in their function and axonal connectivity. First, reliable odor responses of TCs had shorter onset latencies than MCs across odor concentrations. TCs demonstrated odor-induced spike responses with a lower concentration threshold than MCs. Thus, TCs showed reliable responses in a wide range of odor concentration, whereas MCs responded only to high concentration. Second, TCs projected axons densely to focal targets only in anterior areas of the OC, whereas MCs dispersedly projected to nearly all OC areas. In each of the anterior areas that received both MC and TC inputs, the axons of TCs and MCs projected to distinct, largely nonoverlapping parts. These results suggest that TCs convey fast signals with shorter latency to TC-specific targets in the anterior areas of OC, while MCs transmit late-onset signals to the MCspecific targets in the anterior and posterior areas of OC. TCs may convey both weak and strong odor signals, whereas MCs may deliver only strong odor signals. Based on these results, we propose that the axonal projection of $\mathrm{OB}$ neurons to the $\mathrm{OC}$ is com- 

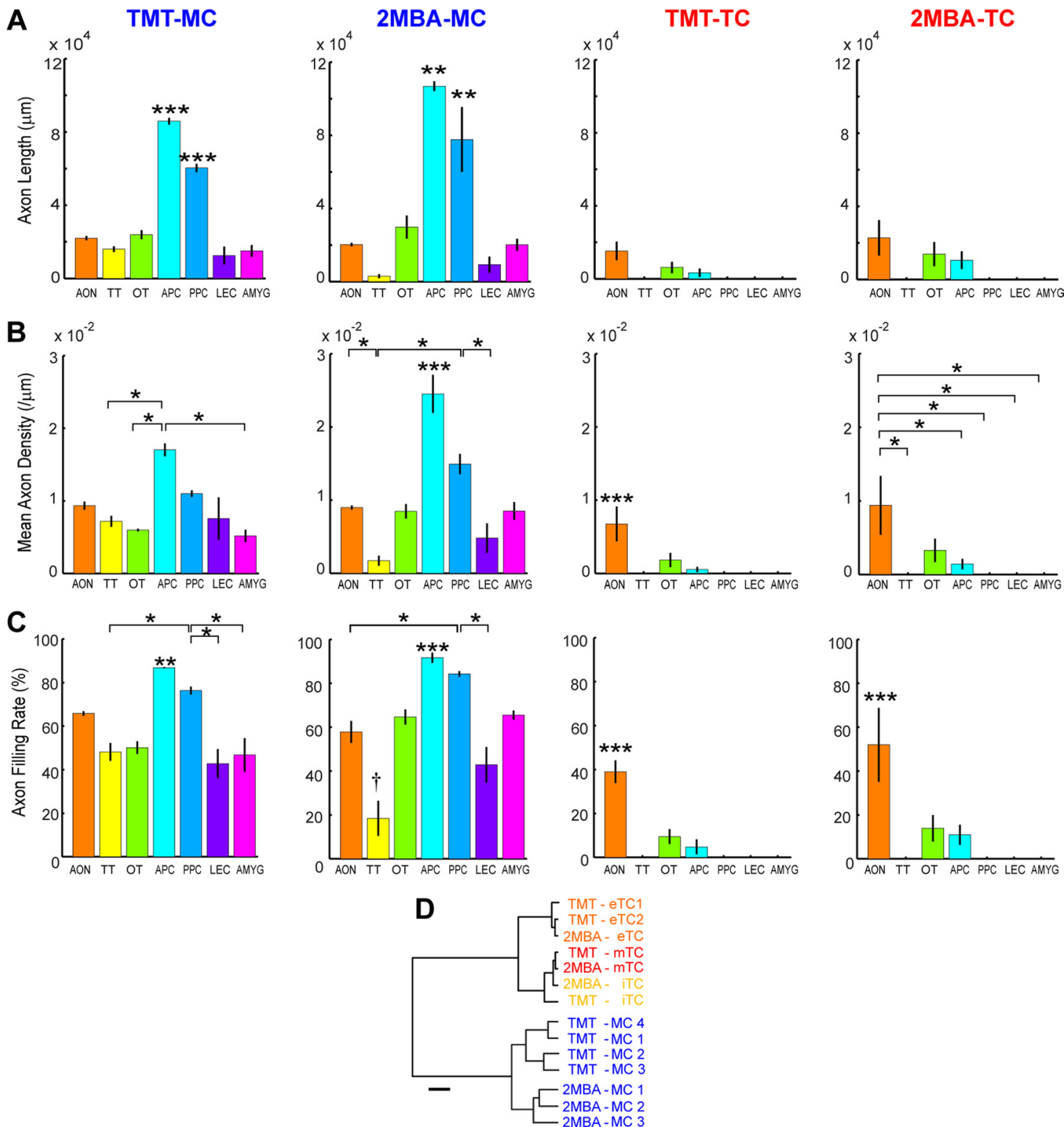

Figure 8. Axonal projection patterns of mitral and tufted cells in individual areas of the olfactory cortex. Total axon length $(\boldsymbol{A})$, mean axon density $(\boldsymbol{B})$, and axon filling rate $(\boldsymbol{C})$ were plotted for TMT-responsive MCs (TMT-MC) (first column), 2MBA-responsive MCs (2MBA-MC) (second column), TMT-responsive TCs (TMT-TC) (third column), and 2MBA-responsive TCs (2MBA-TC) (fourth column). In this analysis, the $\mathrm{OC}$ was divided into seven areas consisting of the anterior olfactory nucleus (AON), tenia tecta (TT), olfactory tubercle (OT), anterior piriform cortex (APC), posterior piriform cortex (PPC), lateral entorhinal cortex (LEC), and olfactory amygdala region (AMYG) including the nucleus of the lateral olfactory tract (nLOT), anterior cortical amygdaloid nucleus (ACO), and posterolateral cortical amygdaloid nucleus (PLCO). The axon filling rate was computed to estimate the area that a given neuron occupied in individual areas of the $0 C$ (see Materials and Methods). Distribution patterns of axons differed across different areas (ANOVA, $p<0.001$ ). Results from Tukey's post hoc HSD test $(p<0.05)$ were shown with a single asterisk. Significant differences from all the other areas are shown with triple asterisks. Double asterisks in the APC or PPC indicate significant difference from all the other areas except from the PPC or APC, respectively. For TMT-MC and 2MBA-MC, the APC had significantly more axonal projections compared with all other areas, and the PPC had more axonal projections compared with three of the other areas. Error bars indicate SEM. D. Cluster analysis of axonal projection patterns of individual MCs and TCs in the areas of the 0 . The hierarchical tree grouped the 14 neurons into two clusters according to the similarity of their axonal projection patterns across the $\mathrm{OC}$ areas. These two clusters coincided with the MC and TC groups. The horizontal bar indicates $200 \mathrm{U}$ in Euclidian distance.

posed of two parallel systems, each of which may have distinct logic of function and connectivity (Fig. 10). The parallel MC and TC pathways may form the functional backbone of the central olfactory circuits.

\section{Functions of fast TC signals and slow MC signals}

Signal timing including response latencies is a crucial parameter of sensory coding in the visual (Gawne et al., 1996; Gollisch and Meister, 2008), somatosensory (Panzeri et al., 2001; Petersen et al., 2001), and auditory (Carr and Konishi, 1990; Heil, 1997) systems. Response latencies may also have significant functional roles in the Xenopus olfactory system (Junek et al., 2010). In the present study, we compared this property in MCs and TCs and found a substantial distinction in response to identical odorants (TMT or 2MBA) and concentrations. TCs had an average onset latency of $\sim 110 \mathrm{~ms}$, whereas MCs had one of $\sim 250-300 \mathrm{~ms}$. Considering the recent results that mice and rats make fast decisions with an odor sampling duration of $150-250 \mathrm{~ms}$ when odor cues are easy to dissociate (Uchida and Mainen, 2003; Rinberg et al., 2006), our results strongly point to a critical role for TCs in this fast response in olfactory behavior. When odor cues become difficult to judge (i.e., in a mixture of similar odors), rodents 
A
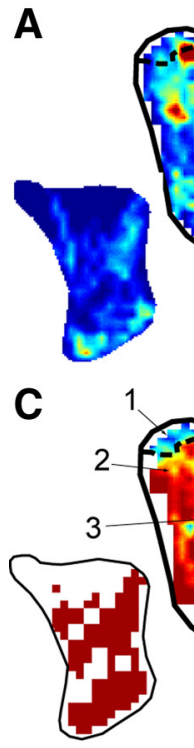

MCs

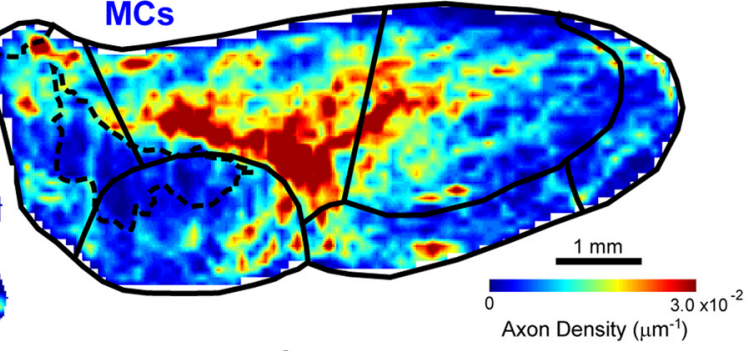

6
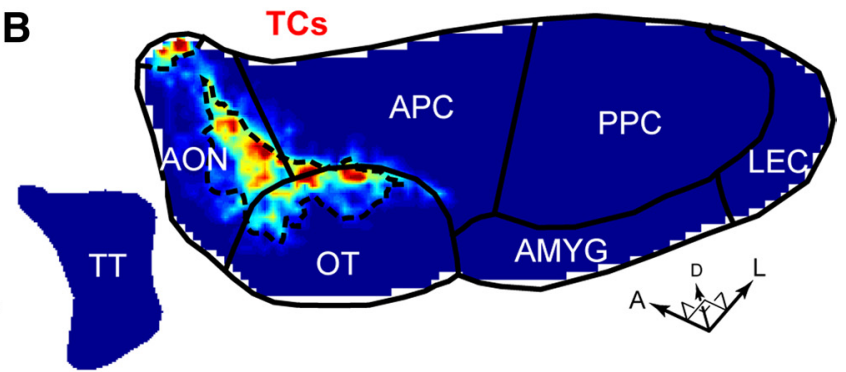

D

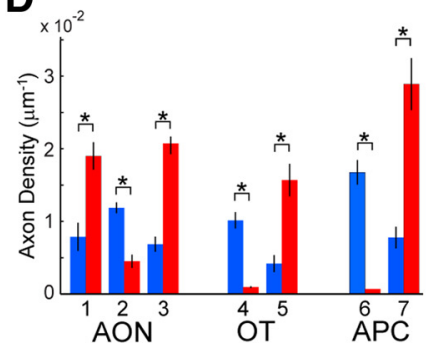

$\mathbf{F}$

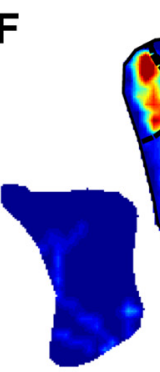

2MBA-MC/TCs

E

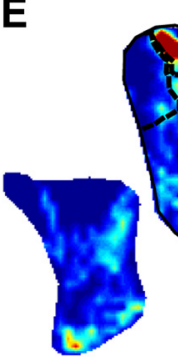

TMT-MC/TCs

G

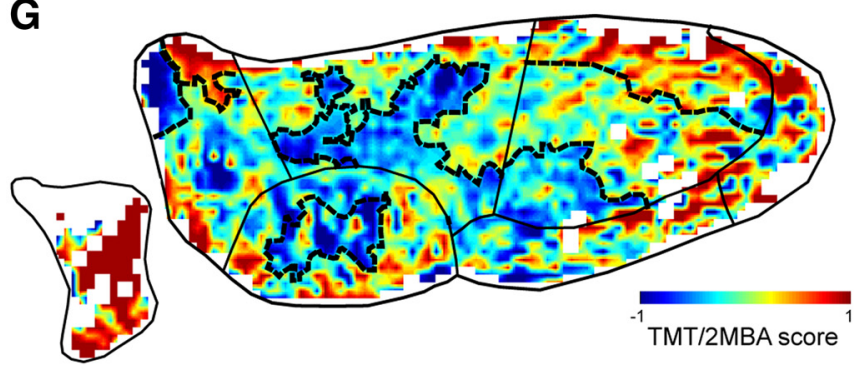

Figure 9. Mitral and tufted cells project axons to segregated subareas in the olfactory cortex. $\boldsymbol{A}, \boldsymbol{B}$, Averaged axon density maps in the $0 \mathrm{C}$ for MCs $(\boldsymbol{A})(n=7$; including $n=4$ TMT-MCs and $n=$ 3 2MBA-MCs) and TCs $(B)(n=7$; including $n=4$ TMT-TCs and $n=3$ 2MBA-TCs). The dotted lines indicate boundaries of MC- and TC-dominant subareas processed from $\boldsymbol{C}$ (see Materials and Methods). The black lines represent the average boundary of the areas in the $\mathrm{OC}$. For simplification, some areas in the $\mathrm{OC}$ are grouped in meta-areas: the anterior olfactory nucleus (AON) including the pars externa and pars principalis (AONpE and AONpP), and olfactory amygdala region (AMYG) including the nucleus of the lateral olfactory tract (nLOT), anterior cortical amygdaloid nucleus (ACO), and posterolateral cortical amygdaloid nucleus (PLCO). APC, Anterior piriform cortex; LEC, lateral entorhinal cortex; 0T, olfactory tubercle; PPC, posterior piriform cortex; TT, tenia tecta; A, anterior; D, dorsal; L, lateral. C, The MC/TC score map in the $0 C$. The MC/TC score $(-1$ to +1$)$ was computed and mapped for each pixel of $100 \mu \mathrm{m}^{2}$. A score of 1 indicates complete MC projection, whereas a score of -1 indicates complete TC projection. The numbers indicate subregions intersected by areal boundaries (black lines) and boundaries of MC- and TC-dominant subareas (dotted line) in the AON (1-3), olfactory tubercle (0T) (4-5), and APC (6-7). $\boldsymbol{D}$, Mean axon densities of MCs (blue bars; $n=7$ ) and TCs (red bars; $n=7)$ in subareas $1-7$ in $\boldsymbol{C} .{ }^{*} p<0.01(t$ test). $\boldsymbol{E}, \boldsymbol{F}$, Averaged axon density maps in the 0 C for TMT-MC/TCS $(\boldsymbol{E})(n=8$; including $n=4$ TMT-MCs and $n=4$ TMT-TCs $)$ and 2MBA-MC/TCs $(\boldsymbol{F})(n=6$; including $n=32$ MBA-MCs and $n=32 \mathrm{MBA}-\mathrm{TCS})$. The dotted lines indicate boundaries between TMT- and 2MBA-dominant subareas processed from $\boldsymbol{G}$. The black lines represent the average boundary of the areas in the $0 C$. $\mathbf{G}$, The TMT/2MBA score map in the OC. A score of 1 indicates complete TMT projection, whereas a score of -1 indicates complete 2MBA projection. $\boldsymbol{H}$, Left, Mean axon densities of TMT-MC/TCs (blue bars; $n=8$ ) and 2MBA-MC/TCs (red bars; $n=6$ ) in subareas a-i. ${ }^{*} p<0.05$; ${ }^{* *} p<0.01$ ( $t$ test). Error bars indicate SEM. Right, The lowercase characters indicate subregions intersected by areal boundaries (black lines) and boundaries of TMT- and 2MBA-dominant subareas (dotted line) in the AON (a, b), tenia tecta (TT) (c), anterior piriform cortex (APC) (d, e), olfactory tubercle (0T) (f), and posterior piriform cortex (PPC) (g-i).

require an additional 100-200 ms sampling time for behavioral accuracy to be maintained (Rinberg et al., 2006). Together with the previous result that MCs have finer odorant discrimination ability than TCs achieved by their finer molecular receptive ranges (Nagayama et al., 2004), the present results suggest that MCs are additionally recruited for difficult odor discrimination. Future studies investigating these roles of TCs and MCs in olfac- tory behaviors using selective inactivation of each cell type will be of great interest.

Synaptic mechanisms for fast TC signals and slow MC signals Recently, patch-clamp recording studies have revealed the direct and indirect synaptic inputs from olfactory sensory neurons (OSNs) to MCs and TCs (Hayar et al., 2004; De Saint Jan et al., 


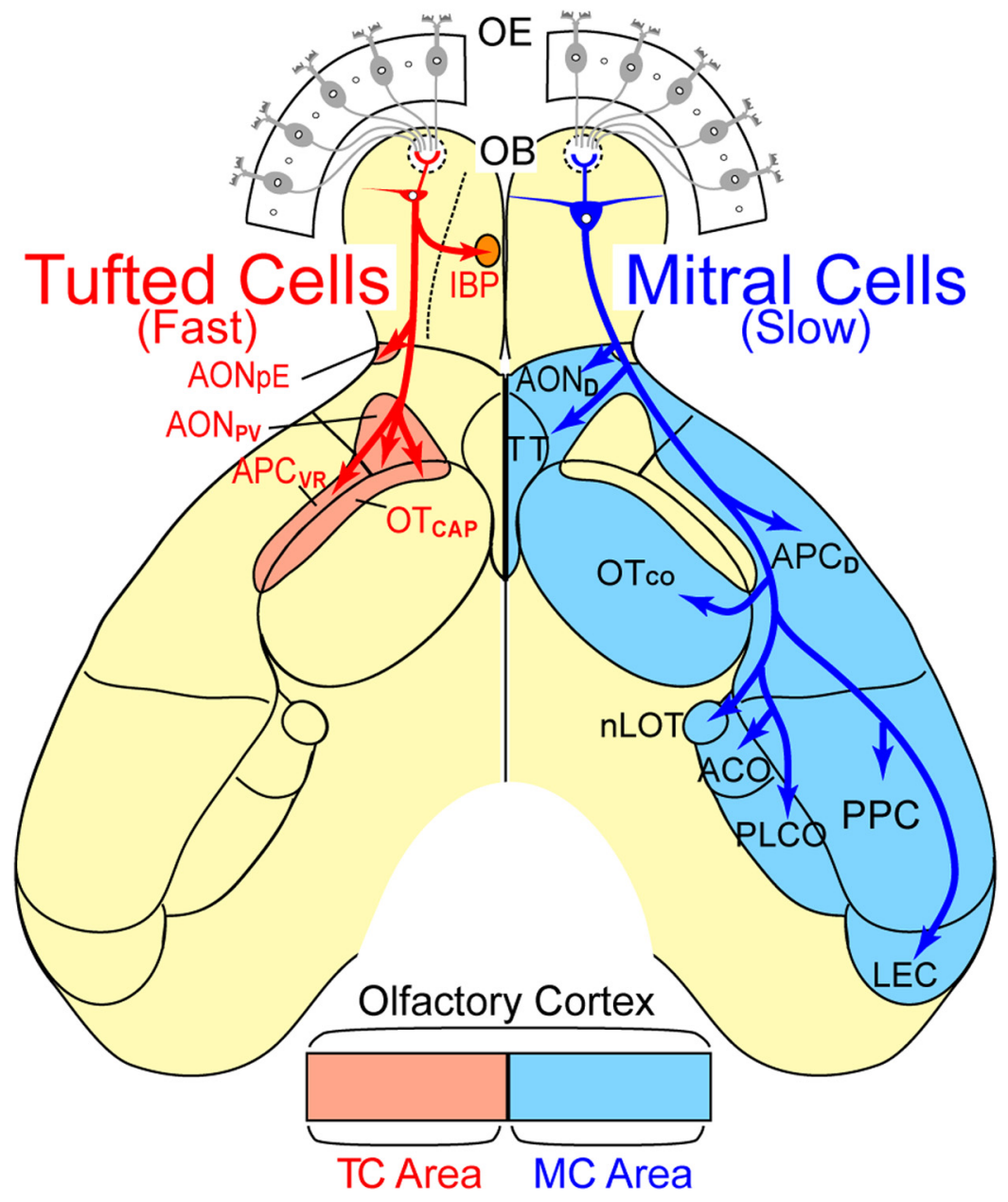

Figure 10. A schematic diagram for parallel pathways of TMT/2MBA-responsive MCs and TCs from the olfactory bulb to the olfactory cortex. The segregated projections of TMT/2MBA-responsive MCs and TCs suggest the existence of parallel MC and TC pathways in the central olfactory system. The axonal projections of the two cell types are represented on the left (TCs, red) and right (MCs, blue) sides of the diagram of the ventral-viewed mouse brain. In the $O C$, TCs route fast odor information to the AON pars externa (AONpE), posteroventral part of the $A O N\left(A O N_{p v}\right)$, anterolateral part of the olfactory tubercle that corresponds to the cap part of the olfactory tubercle $\left(0 \mathrm{~T}_{\mathrm{CAP}}\right)$, and the ventrorostral part of the $\mathrm{APC}\left(\mathrm{APC} \mathrm{VR}_{\mathrm{V}}\right)$. These regions are represented as $\mathrm{TC}$ areas (pink). In the $\mathrm{OB}$, $\mathrm{TCs}$ in the lateral map target the intrabulbar projection axons to the confined small area in the medial map (IBP). By contrast, $M C$ S route slower odor information widely to the dorsal part of the $A O N\left(A O N_{D}\right)$, cortical part of the olfactory tubercle $\left(0 T_{C O}\right)$, dorsal part of the $A P C\left(A P C_{D}\right)$, tenia tecta (TT), posterior piriform cortex (PPC), lateral entorhinal cortex (LEC), nucleus of the lateral olfactory tract (nLOT), anterior cortical amygdaloid nucleus (ACO), and posterolateral cortical amygdaloid nucleus (PLCO) (MC area, cyan). OE, Olfactory epithelium.

2009; Gire and Schoppa, 2009; Najac et al., 2011). The only TC subtype explored to date, eTCs, show strong short-latency firing response to electrical stimulation of OSN axons mediated by the direct excitatory synaptic input from OSN axon terminals on eTCs (De Saint Jan et al., 2009). By contrast, MCs demonstrate short-latency depolarization followed by larger and long-lasting depolarization by a single electrical stimulation of OSN axons. The slow and long-latency component of the depolarization was shown to be an indirect feedforward excitation mediated by intraglomerular dendro-dendritic extrasynaptic inputs from sister TCs and MCs (Carlson et al., 2000; De Saint Jan et al., 2009; Gire and Schoppa, 2009). Stronger electrical stimulation of OSN axons is required to activate the long-latency indirect inputs. Our present result that TCs respond to odor stimulation with a lower concentration threshold and faster onset latency is consistent with these previous observations. In addition, our result that MCs show odor responses only to higher concentrations and with longer onset latency is consistent with above in vitro observations on the large slow component of the depolarization in MCs. These results suggest that the spike output of TCs is shaped mainly by the fast direct synaptic input from OSN axons, which may reflect instantaneous odor signals from the external world. By contrast, the spike output of MCs depends not only on the direct input but more heavily on the slow indirect inputs that may reflect the activity state of sister TCs and MCs (Dhawale et al., 2010). Although the recorded MC population and $\mathrm{mTC}$ population showed a clear difference in response latency and odor concentration threshold, we cannot rule out the possibility that some of the sampled MCs and TCs might be associated with other weakly responsive glomeruli. It will be the task of future studies to further explore functional distinctions of MCs and TCs by comparing MCs and TCs associated with a single glomerulus (Tan et al., 2010).

Target subareas of fast TC signals in the anterior $\mathrm{OC}$ areas

TMT/2MBA-responsive MCs and TCs demonstrated a marked difference in their axonal projection patterns in the OC. Even in the anterior three $\mathrm{OC}$ areas (AON, APC, and olfactory tubercle) that have been previously reported to receive both MC and TC projections (Haberly and Price, 1977; Scott, 1981; Luskin and Price, 1982), MCs and TCs targeted their axons to distinct, largely non-overlapping subareas. Therefore, each of the three areas can be divided into subareas that receive selective axonal inputs either from MCs or TCs (Fig. 10). TCs formed highdensity bushes of axon collaterals in the restricted subarea of the rostroventral APC. This area coincides with the ventralmost portion of the ventrorostral APC $\left(\mathrm{APC}_{\mathrm{VR}}\right)$, which projects heavily to the ventrolateral orbitofrontal cortex (Ekstrand et al., 2001). Although the functional role of the $\mathrm{APC}_{\mathrm{VR}}$ is still unknown, we speculate that the ventral-most part of the $\mathrm{APC}_{\mathrm{VR}}$ transfers fast olfactory signals provided by TCs to the ventrolateral orbitofrontal cortex, which plays a role in multimodality integration of sensory inputs (Ekstrand et al., 2001). In the olfactory tubercle, TC axons projected preferentially to the cap regions of the anterolateral part (Meyer and Wahle, 1986). Although the cap region and cortical region appear to form structural modules in the olfactory tubercle, the functional roles of the two regions are still unknown. Further studies are needed to elucidate the functional roles of the MC input and TC input to the olfactory tubercle in terms of inducing a variety of motivation behaviors such as appetitive motivation behavior and fearful motivation behavior.

TCs are further subdivided into eTCs, mTCs, and iTCs according to differences in the location of cell bodies and dendritic morphology (Macrides and Schneider, 1982; Shepherd et al., 2004). Whether the three TC subtypes have common axonal pro- 
jections or not has been a matter of debate (Belluscio et al., 2002; Lodovichi et al., 2003; Ghosh et al., 2011). In the present study, we observed that (1) all three TC subtypes commonly had intrabulbar projection axons that targeted the mirror symmetric position in the medial map and (2) all three TC subtypes show a distinct axonal projection pattern from those of MCs. These results are in contrast to the observation in a recent study reporting that iTCs and MCs do not have any distinction in their axonal projections (Ghosh et al., 2011). Although this discrepancy may result from their partial labeling of axons with virus staining protocols, further studies with large sampling are required to address this issue.

The glomerular map of mouse OB has two functionally distinct dorsal (D) and ventral (V) domains. The $\mathrm{D}$ domain can be further divided into $\mathrm{D}_{\mathrm{I}}$ and $\mathrm{D}_{\mathrm{II}}$ domains (Kobayakawa et al., 2007; Matsumoto et al., 2010). Specific subsets of glomeruli in the $\mathrm{D}_{\text {II }}$ domain mediate innate fear responses to the predator fox odor TMT, whereas selective subsets of glomeruli in the $\mathrm{D}_{\mathrm{I}}$ domain are involved in aversive responses to the spoiled food odor 2MBA (Kobayakawa et al., 2007), suggesting functional compartmentalization within the OB in terms of odor-induced behavioral responses (Mori and Sakano, 2011). In this study, we focused on the functional and connectivity differences between MCs and TCs, which were observed both for the 2MBA-responsive projection neurons in the $\mathrm{D}_{\mathrm{I}}$ domain and the TMT-responsive projection neurons of the $\mathrm{D}_{\mathrm{II}}$ domain, and noted the functional relevance of the parallel MC and TC pathways in the axonal projection from the $\mathrm{OB}$ to OC. These results suggest that the functional logic of the axonal projection should be explored in each of the MC and TC pathways. Although we noted several difference in the axonal projection patterns between TMT-responsive cells in $\mathrm{D}_{\mathrm{I}}$ domain and 2MBA-responsive cells in $\mathrm{D}_{\mathrm{II}}$ domains, further analyses of the olfactory information processing and axonal projection pattern in each of the MC and TC pathways that are associated with functionally identified glomeruli in the $\mathrm{D}_{\mathrm{I}}, \mathrm{D}_{\mathrm{II}}$, and $\mathrm{V}$ domains of the glomerular map should reveal distinct functional streams of olfactory information processing in the MC pathway and TC pathway. Our approach, which combines functional characterization of spike responses with visualization of long-range axonal circuits at single-cell resolution, will be crucial in this effort.

\section{Notes}

Supplemental material for this article is available at http://www.igaras. net/pub/JNeurosci2012/ and includes 3-D reconstruction movies of a $\mathrm{MC}$ and a TC. This material has not been peer reviewed.

\section{References}

Abraham NM, Spors H, Carleton A, Margrie TW, Kuner T, Schaefer AT (2004) Maintaining accuracy at the expense of speed: stimulus similarity defines odor discrimination time in mice. Neuron 44:865-876.

Bathellier B, Buhl DL, Accolla R, Carleton A (2008) Dynamic ensemble odor coding in the mammalian olfactory bulb: sensory information at different timescales. Neuron 57:586-598.

Belluscio L, Lodovichi C, Feinstein P, Mombaerts P, Katz LC (2002) Odorant receptors instruct functional circuitry in the mouse olfactory bulb. Nature 419:296-300.

Carey RM, Wachowiak M (2011) Effect of sniffing on the temporal structure of mitral/tufted cell output from the olfactory bulb. J Neurosci 31:10615-10626.

Carlson GC, Shipley MT, Keller A (2000) Long-lasting depolarizations in mitral cells of the rat olfactory bulb. J Neurosci 20:2011-2021.

Carr CE, Konishi M (1990) A circuit for detection of interaural time differences in the brainstem of the barn owl. J Neurosci 10:3227-3246.

Cury KM, Uchida N (2010) Robust odor coding via inhalation-coupled transient activity in the mammalian olfactory bulb. Neuron 68:570-585.

De Saint Jan D, Hirnet D, Westbrook GL, Charpak S (2009) External tufted cells drive the output of olfactory bulb glomeruli. I Neurosci 29:2043-2052.

Dhawale AK, Hagiwara A, Bhalla US, Murthy VN, Albeanu DF (2010) Nonredundant odor coding by sister mitral cells revealed by light addressable glomeruli in the mouse. Nat Neurosci 13:1404-1412.

Ekstrand JJ, Domroese ME, Johnson DM, Feig SL, Knodel SM, Behan M, Haberly LB (2001) A new subdivision of anterior piriform cortex and associated deep nucleus with novel features of interest for olfaction and epilepsy. J Comp Neurol 434:289-307.

Feierstein CE, Quirk MC, Uchida N, Sosulski DL, Mainen ZF (2006) Representation of spatial goals in rat orbitofrontal cortex. Neuron 51:495-507.

Furuta T, Kaneko T, Deschênes M (2009) Septal neurons in barrel cortex derive their receptive field input from the lemniscal pathway. J Neurosci 29:4089-4095.

Gawne TJ, Kjaer TW, Richmond BJ (1996) Latency: another potential code for feature binding in striate cortex. J Neurophysiol 76:1356-1360.

Ghosh S, Larson SD, Hefzi H, Marnoy Z, Cutforth T, Dokka K, Baldwin KK (2011) Sensory maps in the olfactory cortex defined by long-range viral tracing of single neurons. Nature 472:217-220.

Gire DH, Schoppa NE (2009) Control of on/off glomerular signaling by a local GABAergic microcircuit in the olfactory bulb. J Neurosci 29:13454-13464.

Gollisch T, Meister M (2008) Rapid neural coding in the retina with relative spike latencies. Science 319:1108-1111.

Griff ER, Mafhouz M, Chaput MA (2008) Comparison of identified mitral and tufted cells in freely breathing rats: II. Odor-evoked responses. Chem Senses 33:793-802.

Haberly LB, Price JL (1977) The axonal projection patterns of the mitral and tufted cells of the olfactory bulb in the rat. Brain Res 129:152-157.

Hayar A, Karnup S, Shipley MT, Ennis M (2004) Olfactory bulb glomeruli: external tufted cells intrinsically burst at theta frequency and are entrained by patterned olfactory input. J Neurosci 24:1190-1199.

Heil P (1997) Auditory cortical onset responses revisited. II. Response strength. J Neurophysiol 77:2642-2660.

Heimer L (1968) Synaptic distribution of centripetal and centrifugal nerve fibres in the olfactory system of the rat. An experimental anatomical study. J Anat 103:413-432.

Johnson DM, Illig KR, Behan M, Haberly LB (2000) New features of connectivity in piriform cortex visualized by intracellular injection of pyramidal cells suggest that "primary" olfactory cortex functions like "association" cortex in other sensory systems. J Neurosci 20:6974-6982.

Junek S, Kludt E, Wolf F, Schild D (2010) Olfactory coding with patterns of response latencies. Neuron 67:872-884.

Kobayakawa K, Kobayakawa R, Matsumoto H, Oka Y, Imai T, Ikawa M, Okabe M, Ikeda T, Itohara S, Kikusui T, Mori K, Sakano H (2007) Innate versus learned odour processing in the mouse olfactory bulb. Nature 450:503-508.

Konishi M (2003) Coding of auditory space. Annu Rev Neurosci 26:31-55.

Kozloski J, Hamzei-Sichani F, Yuste R (2001) Stereotyped position of local synaptic targets in neocortex. Science 293:868-872.

Lodovichi C, Belluscio L, Katz LC (2003) Functional topography of connections linking mirror-symmetric maps in the mouse olfactory bulb. Neuron 38:265-276.

Luskin MB, Price JL (1982) The distribution of axon collaterals from the olfactory bulb and the nucleus of the horizontal limb of the diagonal band to the olfactory cortex, demonstrated by double retrograde labeling techniques. J Comp Neurol 209:249-263.

Macrides F, Schneider SP (1982) Laminar organization of mitral and tufted cells in the main olfactory bulb of the adult hamster. J Comp Neurol 208:419-430.

Matsumoto H, Kobayakawa K, Kobayakawa R, Tashiro T, Mori K, Sakano H, Mori K (2010) Spatial arrangement of glomerular molecular-feature clusters in the odorant-receptor class domains of the mouse olfactory bulb. J Neurophysiol 103:3490-3500.

Meyer G, Wahle P (1986) The olfactory tubercle of the cat. I. Morphological components. Exp Brain Res 62:515-527.

Miyamichi K, Amat F, Moussavi F, Wang C, Wickersham I, Wall NR, Taniguchi H, Tasic B, Huang ZJ, He Z, Callaway EM, Horowitz MA, Luo L (2011) Cortical representations of olfactory input by trans-synaptic tracing. Nature 472:191-196. 
Mori K, Sakano H (2011) How is the olfactory map formed and interpreted in the mammalian brain? Annu Rev Neurosci 34:467-499.

Mori K, Kishi K, Ojima H (1983) Distribution of dendrites of mitral, displaced mitral, tufted, and granule cells in the rabbit olfactory bulb. J Comp Neurol 219:339-355.

Nagayama S, Takahashi YK, Yoshihara Y, Mori K (2004) Mitral and tufted cells differ in the decoding manner of odor maps in the rat olfactory bulb. J Neurophysiol 91:2532-2540.

Nagayama S, Enerva A, Fletcher ML, Masurkar AV, Igarashi KM, Mori K, Chen WR (2010) Differential axonal projection of mitral and tufted cells in the mouse main olfactory system. Front Neural Circuits 4:pii:120.

Najac M, De Saint Jan D, Reguero L, Grandes P, Charpak S (2011) Monosynaptic and polysynaptic feedforward inputs to mitral cells from olfactory sensory neurons. J Neurosci 31:8722-8729.

Nassi JJ, Callaway EM (2009) Parallel processing strategies of the primate visual system. Nat Rev Neurosci 10:360-372.

Ojima H, Mori K, Kishi K (1984) The trajectory of mitral cell axons in the rabbit olfactory cortex revealed by intracellular HRP injection. J Comp Neurol 230:77-87.

Panzeri S, Petersen RS, Schultz SR, Lebedev M, Diamond ME (2001) The role of spike timing in the coding of stimulus location in rat somatosensory cortex. Neuron 29:769-777.

Petersen CC (2007) The functional organization of the barrel cortex. Neuron 56:339-355.

Petersen RS, Panzeri S, Diamond ME (2001) Population coding of stimulus location in rat somatosensory cortex. Neuron 32:503-514.

Pinault D (1996) A novel single-cell staining procedure performed in vivo under electrophysiological control: morpho-functional features of juxtacellularly labeled thalamic cells and other central neurons with biocytin or Neurobiotin. J Neurosci Methods 65:113-136.

Price JL (1973) An autoradiographic study of complementary laminar patterns of termination of afferent fibers to the olfactory cortex. J Comp Neurol 150:87-108.
Rinberg D, Koulakov A, Gelperin A (2006) Speed-accuracy tradeoff in olfaction. Neuron 51:351-358.

Rubin BD, Katz LC (1999) Optical imaging of odorant representations in the mammalian olfactory bulb. Neuron 23:499-511.

Schoenfeld TA, Marchand JE, Macrides F (1985) Topographic organization of tufted cell axonal projections in the hamster main olfactory bulb: an intrabulbar associational system. J Comp Neurol 235:503-518.

Scott JW (1981) Electrophysiological identification of mitral and tufted cells and distributions of their axons in olfactory system of the rat. J Neurophysiol 46:918-931.

Shepherd GM, Chen WR, Greer CA (2004) Olfactory bulb. In: The synaptic organization of the brain (Shepherd GM, ed), pp 165-216. Oxford: Oxford UP.

Shepherd GM, Stepanyants A, Bureau I, Chklovskii D, Svoboda K (2005) Geometric and functional organization of cortical circuits. Nat Neurosci 8:782-790.

Sosulski DL, Bloom ML, Cutforth T, Axel R, Datta SR (2011) Distinct representations of olfactory information in different cortical centres. Nature 472:213-216.

Tan J, Savigner A, Ma M, Luo M (2010) Odor information processing by the olfactory bulb analyzed in gene-targeted mice. Neuron 65:912-926.

Uchida N, Mainen ZF (2003) Speed and accuracy of olfactory discrimination in the rat. Nat Neurosci 6:1224-1229.

Uchida N, Takahashi YK, Tanifuji M, Mori K (2000) Odor maps in the mammalian olfactory bulb: domain organization and odorant structural features. Nat Neurosci 3:1035-1043.

Wachowiak M (2011) All in a sniff: olfaction as a model for active sensing. Neuron 71:962-973.

Wong AM, Wang JW, Axel R (2002) Spatial representation of the glomerular map in the Drosophila protocerebrum. Cell 109:229-241.

Yoshida I, Mori K (2007) Odorant category profile selectivity of olfactory cortex neurons. J Neurosci 27:9105-9114. 